\title{
fluage héréditaire et consolidation d'un sol quasi-saturé
}

\author{
par \\ B. Félix \\ Ingénieur au Département des Sols et fondations du Laboratoire Central des Ponts et Chaussées
}

La théorie du fluage héréditaire fournit une description correcte du fluage des sols fins soumis, en compression codométrique, à des charges constantes ou progressivement croissantes (Félix, $1980 \mathrm{c}$ ). Dans la formulation mathématique de la consolidation unidimensionnelle présentée dans cet article, son équation d'état est utilisée pour décrire la déformation du squelette intergranulaire. Cette formulation répond à deux préoccupations.

II s'agit tout d'abord de rendre compte des principales divergences observées entre les mesures effectuées au laboratoire ou sous les ouvrages dans des conditions de drainage et de déformation unidimensionnelles, et les résultats de la théorie de la consolidation de K. Terzaghi (1923). Ces divergences concernent le développement dans le temps de la déformation, (en particulier l'apparition d'une consolidation secondaire) et les valeurs maximales des surpressions interstitielles, ainsi que la durée de leur dissipation.

D'autre part, la formulation mathématique doit recourir à des paramètres de comportement du sol faciles à mesurer, et aboutir à une solution dont le calcul est simple et rapide. C'est à ces deux qualités que la théorie classique de K. Terzaghi doit d'être, encore aujourd'hui, universellement employée. Cet article se divise par conséquent en deux parties. Dans la première, il s'agit de trouver une explication aux divergences évoquées ci-dessus. Les hypothèses posées sont relativement complexes. Par contre dans la deuxième, des simplifications leur sont apportées de façon à formuler le problème de la consolidation en variables réduites et aboutir à des résultats sous forme d'abaques.

\section{Première partie: Résolution du problème de la consolidation unidimensionnelle}

\section{Formulation des équations de la consolida- tion}

Le sol, qui contient des éléments minéraux, liquides et gazeux, est représenté comme la combinaison étroite de deux phases: le squelette intergranulaire et le liquide interstitiel. La formulation mathématique est basée sur leurs équations d'état complétées par les équations de continuité et d'équilibre; les déformations des deux phases sont identiques, et la somme des contraintes qui leur sont appliquées est en équilibre avec les charges extérieures.

Les modifications apportées à la théorie de la consolidation de $\mathrm{K}$. Terzaghi concernent les deux équations d'état et la prise en compte des grandes déformations.

\subsection{Les grandes déformations}

La consolidation d'un sol de fondation peut se traduire par des déformations supérieures à $20 \%$; par conséquent, la distance d'un plan quelconque au substratum imperméable, égale à z au moment du chargement, est représentée au temps $t$, par une fonction $s(z, t)$ qui est liée à la déformation correspondante par la relation (1):

$$
\varepsilon(z, t)=\lim _{\Delta z \rightarrow 0} \frac{\Delta z-\Delta s(z, t)}{\Delta z}=1-\frac{\partial s(z, t)}{\partial z} .
$$

Si h est l'épaisseur initiale du monocouche, sa déformation moyenne est égale au temps $\mathrm{t}$ à :

$$
\varepsilon(z, t)=\frac{1}{h} \int_{0}^{h} \varepsilon(z, t) d z=\frac{h-s(h, t)}{h} \text {. }
$$




\section{2 Équation d'état du squelette intergranulaire}

L'empilement des particules minérales entourées d'eau adsorbée est assimilé à un corps visco-élastique non linéaire, dont la déformation sous une contrainte effective variant dans le temps est égale à :

$$
\varepsilon(t)=\int_{0}^{t} K(t-\tau) f\left[\sigma^{\prime}(\tau)\right] d \tau .
$$

Cette équation d'état est déduite de la théorie du fluage héréditaire non linéaire, en supposant que la déformation instantanée est nulle (Félix, $1980 \mathrm{c}$ ).

$K(t)$ est égal à la vitesse de fluage sous une contrainte effective constante, égale à la charge extérieure appliquée $\sigma_{1}^{\prime}$, après une durée d'application t. La primitive, la déformation elle-même, est représentée par la fonction (4) appelée mesure de fluage:

$$
C(t)=\varepsilon_{t}\left(1-\exp \left(-\alpha t^{\beta}\right)\right)
$$

où $\varepsilon_{\text {, }}$ est la déformation finale atteinte au bout d'un temps infini. Les paramètres $\alpha$ et $\beta$ sont déterminés très simplement à partir des déformations de consolidation secondaire mesurées à l'œdomètre (Félix, $1980 \mathrm{c}$ ).

$f\left(\sigma^{\prime}\right)$ est une fonction non linéaire de la contrainte, égale à 1 pour la contrainte extérieure $\sigma_{1}$ appliquée au cours de la consolidation et à 0 pour la contrainte initiale avant chargement $\sigma_{0}$.

$f\left(\sigma^{\prime}\right)$ est appelée fonction de contrainte. Elle est continue et définie par les deux relations (5) qui caractérisent respectivement les comportements surconsolidé et normalement consolidé du squelette :

$$
\begin{array}{ll}
\sigma^{\prime}<\sigma_{p}^{\prime} & f\left(\sigma^{\prime}\right)=a_{1} \lg \sigma^{\prime}-b_{1} \\
\sigma^{\prime}>\sigma_{p}^{\prime} & f\left(\sigma^{\prime}\right)=a_{2} \lg \sigma^{\prime}-b_{2}
\end{array}
$$

avec

$$
a_{1} \lg \sigma_{p}^{\prime}-b_{1}=a_{2} \lg \sigma_{p}^{\prime}-b_{2}
$$

où $\sigma_{p}^{\prime}$ est la contrainte de préconsolidation.

Les paramètres $a_{1}, b_{1}, a_{2}, b_{2}$ sont calculés à partir de $\sigma_{p}^{\prime}$ et des déformations de consolidation secondaire mesurées sous $\sigma_{1}$ et sous une deuxième contrainte intermédiaire $\sigma_{2}$.

II est possible de les déterminer approximativement grâce aux résultats d'un essai classique de compressibilité à l'œdomètre (Félix, 1980 c).

\section{3 Équation d'état du liquide interstitiel}

II est très fréquent de rencontrer en place des sols mous imparfaitement saturés. Le gaz qu'ils contiennent, sous forme de bulles occluses ou en solution dans l'eau, provient de la décomposition des matières organiques ou de l'air atmosphérique, si le sol est situé au-dessus du niveau phréatique.

Si le degré de saturation excède $85 \%$, les bulles restent disjointes (Schuurman, 1966) et le liquide interstitiel peut être considéré comme homogène, mais doté d'une compressibilité apparente. Le sol est alors quasi-biphasique. Cette compressibilité qui a une grande influence sur le développement de la consolidation, a été étudiée à drainage fermé par Schuurman (1966), Ter Martirosyan (1967), Fredlund (1976), Magnan et Dang (1977).

Lorsque la pression interstitielle définie par son excès sur la pression atmosphérique $P_{0}$, passe de $u_{0}$ à $u_{\text {, la }}$ variation relative de volume par unité de pression est égale, d'après Magnan et Dang (1977) à :

$$
a_{w}^{o}=\frac{V_{w}^{o}-V_{w}}{V_{w}^{0}} \frac{1}{u-u_{0}}=\frac{1-S_{r}^{o}(1-H)}{u+P_{o}+S_{r} \frac{2 q}{r}} .
$$

L'indice 0 est relatif à l'état initial du fluide; $V_{w}$ est le volume du liquide interstitiel;

$\mathrm{S}_{\mathrm{r}}$ son degré de saturation;

$\mathrm{H}$ le coefficient de solubitité du gaz dans l'eau (coefficient de Henry);

$q$ est le coefficient de tension superficielle de l'eau pure et $r$ le rayon moyen des bulles de gaz.

Les pressions sont mesurées en $\mathrm{kPa}$. Le terme $\mathrm{S}_{\mathrm{r}} \frac{2 q}{\mathrm{r}}$ est supposé constant pour les valeurs courantes de la pression interstitielle, et égal à 30 . La pression atmosphérique est égale à 100 .

Si la pression interstitielle initiale $u_{0}$ est nulle, la compressibilité apparente de la phase liquide sous une pression $\mathrm{u}$ est liée à $\mathrm{a}_{\mathrm{w}}^{\circ}$ de la manière suivante :

$$
C_{w}=\frac{1}{V_{w}^{0}} \frac{d V_{w}}{d u}=\frac{d\left(a_{w}^{0} u\right)}{d u}=\frac{130\left(1-S_{r}^{0}(1-H)\right)}{(u+130)^{2}} .
$$

Au cours de la consolidation la phase liquide occupe un volume décroissant. Si le gaz et le liquide s'échappent en proportion constante, le degré de saturation ne varie pas et la compressibilité est toujours définie par la formule (8).

Au moment du chargement, la phase liquide occupe une fraction $n_{0}$ du volume, égale à la porosité du sol dans son état initial.

Au temps $t$ après le chargement et en un point de cote initiale $z$, cette fraction du volume total initial est devenue : $n_{0}-\varepsilon(z, t)$,

si l'on suppose les particules minérales incompressibles.

La déformation par unité de temps de la phase liquide, due à la variation de la pression interstitielle est égale à :

$$
\frac{\partial u(z, t)}{\partial t} C_{w}(z, t)\left(n_{0}-\varepsilon(z, t)\right)
$$

et celle provoquée par le flux de liquide à travers les pores du sol à

$$
\operatorname{div} \overrightarrow{\mathrm{V}}=\frac{\partial \mathrm{V}}{\partial \mathrm{z}}
$$

où $\mathrm{V}$ est la vitesse par rapport au squelette, du fluide interstitiel équivalent qui occuperait le volume total du sol. Elle est liée au gradient des surpressions interstitielles par la loi de Darcy

$$
v=-\frac{1}{\gamma_{w}} k(z, t) \frac{\partial u}{\partial s}
$$

$\gamma_{w}$ est le poids volumique de l'eau,

$s$ est la valeur au temps $I$ de la cote initiale $z$ et $k(z, t)$ le coefficient de perméabilité du sol qui est lié à l'indice des vides $e(z, t)$ par la relation classique:

$$
e(z, t)=A+B \lg k(z, t)
$$

et donc à la déformation par

$$
k(z, t)=\exp \left(A^{\prime}+B^{\prime} \varepsilon(z, t)\right)
$$

où $A^{\prime}=\frac{e_{0}-A}{B} \ln 10$ et $B^{\prime}=-\frac{\left(1+e_{0}\right)}{B} \ln 10$.

L'équation d'état du liquide interstitiel exprime que la déformation de cette phase est égale à la somme des termes (9) et (10).

$$
\begin{aligned}
\frac{\partial \varepsilon(z, t)}{\partial t}=\frac{\partial u(z, t)}{\partial t} c_{w}(z, t)\left[n_{0}\right. & -\varepsilon(z, t)] \\
& -\frac{1}{\gamma_{w}} \frac{\partial}{\partial z}\left[k(z, t) \frac{\partial u}{\partial z}\right]
\end{aligned}
$$




\subsection{Système d'équations à résoudre}

La contrainte extérieure appliquée reste égale, au cours de la consolidation, à $\sigma_{1}$. L'équation d'équilibre (15) :

$$
\sigma_{1}=\sigma^{\prime}+\mathrm{u}
$$

permet de substituer, dans l'équation d'état (14), la contrainte effective à la surpression interstitielle. L'équation de continuité, c'est-à-dire l'égalité des vitesses de déformation des deux phases, conduit à l'équation de la consolidation suivante:

$$
\begin{array}{r}
\frac{\partial}{\partial t}\left[\int_{0}^{t} K(t-\tau) f\left[\sigma^{\prime}(z, \tau)\right] d \tau\right]=\frac{1}{\gamma_{w}} \frac{\partial}{\partial z}\left[k(z, t) \frac{\partial \sigma^{\prime}(z, t)}{\partial s}\right] \\
-\frac{\partial \sigma^{\prime}(z, t)}{\partial t} C_{w}(z, t)\left[n_{0}-\varepsilon(z, t)\right] .
\end{array}
$$

\section{Résolution numérique par le programme de calcul Hydroflu}

La résolution est effectuée suivant la méthode des différences finies.

\subsection{Les variables de temps et d'espace}

La valeur numérique des fonctions « contrainte effective" et "déformation" ainsi que celle des paramètres variables (perméabilité et compressibilité du liquide interstitiel) est calculée en une série de n plans équidistants parallèles au substratum imperméable, et pour des valeurs du temps séparées par des intervalles croissant suivant une progression géométrique (fig. 1).

$$
\begin{gathered}
t_{i+1}-t_{i}=D \cdot r^{j-1} \quad \text { avec } r>1 \\
t_{i+1}=D \cdot\left(\sum_{k=1}^{i-1} r^{k}\right)=\frac{D\left(r^{i}-1\right)}{r-1} \simeq \frac{D r^{i}}{r-1}
\end{gathered}
$$

quand $\mathrm{i}$ est grand.

Le premier intervalle de durée D est choisi suffisamment petit pour que la variation des solutions $\sigma^{\prime}$ et $\varepsilon$ soit modérée sur cet intervalle, malgré les grandes vitesses initiales de fluage du squelette et de compression de la phase liquide. A la fin du calcul, le pas de temps défini par (17) est très grand mais la précision du calcul reste correcte car les contraintes effectives sont égales à la charge extérieure appliquée et la déformation du sol évolue lentement suivant le fluage du squelette. Cette répartition des valeurs du temps a l'avantage de réduire considérablement la quantité de calcul nécessaire pour simuler la consolidation d'un monocouche. Nous verrons également qu'elle en présente d'autres.

\subsection{Formulation en différences finies}

Sur chaque intervalle de temps $t_{i}, t_{i+1}$, la contrainte

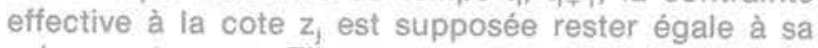
valeur au temps $t_{i}$. Elle progresse, par conséquent, par paliers et la déformation qu'elle provoque s'écrit au temps $\mathrm{t}_{\mathrm{i}+1}$ (formule (35), Félix, $1980 \mathrm{c}$ ) :

$\varepsilon_{i, i+1}=f\left[\sigma_{j}^{\prime}, 1\right] C\left(t_{i+1}\right)+\sum_{k=1}^{i}\left[f\left[\sigma_{i, k+1}^{\prime}\right]-f\left[\sigma_{j, k}^{\prime}\right] C\left(t_{i+1}-t_{k}\right)\right.$,

la vitesse moyenne de déformation, sur ce même intervalle de temps, étant de ce fait égale à :

Fig. 1 Schéma de principe de la résolution par différences finies

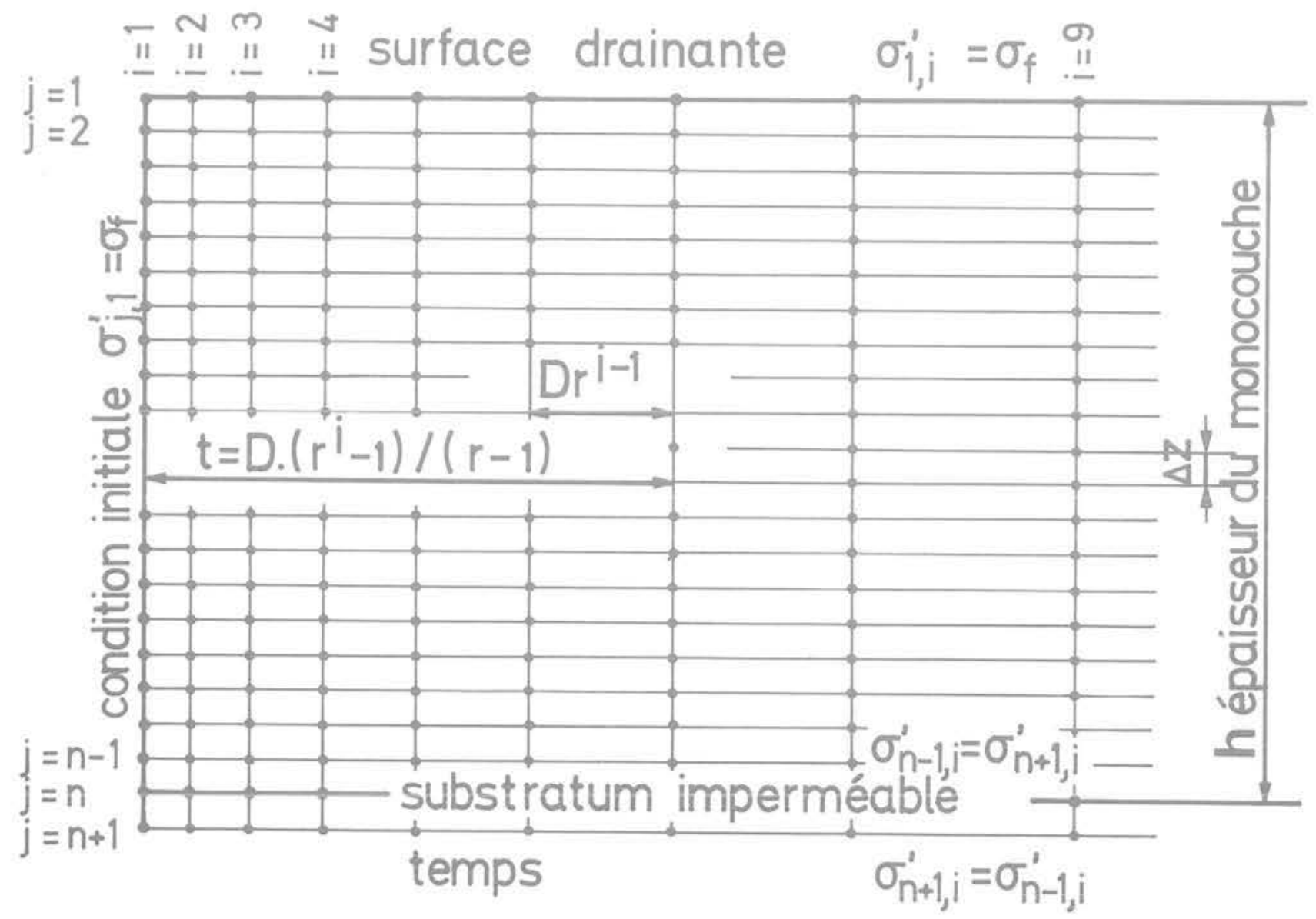




$$
\begin{aligned}
& \frac{\varepsilon_{1,1+1}-\varepsilon_{j, i}}{t_{i+1}-t_{i}}=\frac{1}{t_{i+1}-t_{i}}\left\{f\left(\sigma_{i, 1}^{\prime}\right)\left[C\left(t_{i+1}\right)-C\left(t_{i}\right)\right]\right. \\
& \left.+\sum_{k=1}^{i}\left[f\left(\sigma_{j, k+1}^{\prime}\right)-f\left(\sigma_{i, k}^{\prime}\right)\right]\left[C\left(t_{i+1}-t_{k}\right)-C\left(i_{i}-t_{k}\right)\right]\right\} .
\end{aligned}
$$

La perméabilité moyenne au-dessus et en dessous de ce plan, respectivement $k_{i, l}^{+}$et $k_{i, j}^{-}$est calculée en supposant que la variation de ce paramètre est localement représentée par une fonction parabolique de la cote $z$ initiale :

et

$$
k_{j, 1}^{+}=\frac{3}{8} k_{i+1,1}+\frac{3}{4} k_{i, j}-\frac{1}{8} k_{j-1,1}
$$

$$
k_{j, i}^{-}=-\frac{1}{8} k_{j+1,1}+\frac{3}{4} k_{j, i}+\frac{3}{8} k_{j-1, i}
$$

$k_{j-1, i,} k_{j, j}$ et $k_{j+1, j}$ étant égaux à la perméabilité au temps $t_{1}$ dans les plans des cotes $z_{i-1}, z_{j}$ et $z_{i+1}$ respectivement. $\left(\Delta z=z_{j+1}-z_{j}=z_{i}-z_{j-1}\right.$.)

La vitesse de déformation de la phase liquide décrite par l'expression (14) devient en différences finies:

$$
\begin{array}{r}
\frac{\varepsilon_{j, i+1}-\varepsilon_{i, j}}{t_{i+1}-t_{i}}=\frac{1}{\gamma_{w} \Delta z}\left[\frac{k_{j, i}^{-}\left(\sigma_{i-1, i}^{\prime}-\sigma_{j, i}^{\prime}\right)}{\left(1-\varepsilon_{j, i}\right) \Delta z}-\frac{k_{j, i}^{+}\left(\sigma_{i, i}^{\prime}-\sigma_{i+1, i}^{\prime}\right)}{\left(1-\varepsilon_{j, i}\right) \Delta z}\right] \\
\quad-\frac{\sigma_{j, i+1}^{\prime}-\sigma_{i, i}^{\prime}}{t_{i+1}-t_{i}} \frac{130\left(1-S_{r}^{0}(1-H)\right)}{\left(\sigma_{1}-\sigma_{j, i}^{\prime}+130\right)^{2}}\left(n_{0}-\varepsilon_{i, i}\right) . \quad \text { (20) }
\end{array}
$$

\subsection{Matrice d' "histoire des contraintes"}

Lorsque le calcul a atteint la $i+1^{i \text { iome }}$ itération, l'équation de la consolidation, qui est l'égalité des termes (18) et (20) ne contient qu'une seule inconnue $\sigma_{i, i+1}^{\prime}$ qui est fonction des trois contraintes $\sigma_{j-1, i}^{\prime}, \sigma_{i, i}^{\prime}$ et $\sigma_{i+1,1}^{\prime}$ dont dépend la valeur du gradient hydraulique, et de l'ensemble des solutions $\sigma_{i, k}^{\prime}$ calculées dans le plan de cote $z_{1}$ jusqu'au temps $t_{1}$ : l'équation d'état de la théorie du fluage héréditaire prend en compte "l'histoire du chargement".

La progression géométrique des intervalles de temps permet de ne faire entrer dans le calcul que les $\mathrm{m}$ dernières contraintes $\sigma_{1, k}^{\prime}$ calculées pour des valeurs du temps comprises entre $t_{p}$ et $t_{i+1}(i-p+1=m)$; d'après (17):

$$
\frac{t_{i+1}}{t_{p}}=\frac{r^{i}-1}{r^{p-1}-1} \approx r^{m}
$$

La déformation différée que provoque au temps $t_{i+1}$ le chargement antérieur à $t_{p}$ et qui est négligée, est inférieure à :

$$
\Delta \varepsilon=K\left(t_{i+1}\right) f\left(\sigma_{i}^{\prime}\right) t_{p} \text {, où } f\left(\sigma_{1}^{\prime}\right)=1,
$$

car $\sigma_{1}^{\prime}$, la charge extérieure, est un majorant des contraintes effectives réelles.

$$
\Delta \varepsilon=\frac{\varepsilon_{q} \alpha \cdot t_{i+1}^{\beta}}{r^{m}} \exp \left(-\alpha t_{i+1}^{\beta}\right) \leqslant \frac{\varepsilon_{t} t_{i+1}^{\beta}}{r^{m}} .
$$

Pour s'assurer que l'erreur commise reste inférieure à une valeur $\Delta \varepsilon$ choisie, il suffit que le nombre de solutions intervenant à chaque pas de temps dans le calcul, soit $\mathrm{m}$, satisfasse à l'inégalité :

$$
m>\frac{\lg \left(\varepsilon_{1} \cdot \alpha \cdot t_{1}^{\beta}\right)-\lg \Delta \varepsilon}{\lg r}
$$

( $t$, valeur maximale du temps marquant la fin du calcul). En pratique, un calcul mené avec une raison $r=1,25$ sur le temps, qui permet en 100 itérations de simuler 20 ans de la consolidation d'un monocouche, tolère une erreur de 0,1 pour cent sur la déformation si $\mathrm{m}=20$.

\subsection{Critère de convergence}

Pour chaque indice $i$ et $\mathrm{j}$ la résolution de l'équation de la consolidation permet de déterminer la contrainte effective $\sigma_{1, i+1}^{\prime}$ et par conséquent $\varepsilon_{1,1+1}$. Cette équation est linéarisée en substituant dans le dernier terme de la sommation (18).

$$
\left(\sigma_{1,1+1}^{\prime}-\sigma_{i, 1}^{\prime}\right) f^{\prime}\left(\sigma_{j, 1}^{\prime}\right) \text { à } f\left(\sigma_{i, i+1}^{\prime}\right)-f\left(\sigma_{j, 1}^{\prime}\right) \text {. }
$$

Ce mode de résolution est explicite. La condition de convergence est analogue à celle de l'équation de la chaleur, de même forme que l'équation de la théorie de $K$. Terzaghi. Les intervalles de temps $t_{i+1}-t_{i}$ doivent rester inférieurs à une borne fonction des paramètres de l'équation, condition incompatible avec la progression géométrique adoptée.

Par conséquent le mode de résolution implicite a été choisi; toutes les inconnues d'indice j sont liées entre elles, et leur détermination passe par la résolution d'un système de n équations de la consolidation, de la forme (22). A cet effet le gradient hydraulique est supposé égal, au cours de chaque intervalle de temps, à la moyenne arithmétique de ses valeurs aux extrémités de cet intervalle.

$$
\begin{aligned}
& \varepsilon_{i, i+1}-\varepsilon_{i, i}=f\left(\sigma_{i, 1}^{\prime}\right)\left(C\left(t_{i+1}\right)-C\left(t_{i}\right)\right)+ \\
& \sum_{k=1}^{i-1}\left(f\left(\sigma_{i, k+1}^{\prime}\right)-f\left(\sigma_{i, k}^{\prime}\right)\right)\left(C\left(t_{i+1}-t_{k}\right)-C\left(t_{i}-t_{k}\right)\right)+ \\
& \left(\sigma_{j, i+1}^{\prime}-\sigma_{i, i}^{\prime}\right) f^{\prime}\left(\sigma_{i, i}^{\prime}\right) C\left(t_{i+1}-t_{i}\right) \\
& \varepsilon_{j, i+1}-\varepsilon_{j, i}=\frac{t_{i+1}-t_{i}}{2 \gamma_{w} \Delta z^{2}\left[1-\varepsilon_{i, j}\right]} \\
& {\left[\sigma_{j-1, i}^{\prime} k_{j, j}^{-}-\sigma_{i, i}^{\prime}\left(k_{j, i}^{-}+k_{j, i}^{+}\right)+\sigma_{j+1,}^{\prime} k_{j, i}^{+}\right.} \\
& \left.+\sigma_{i-1, i+1}^{\prime} k_{i, i}^{-}-\sigma_{i, i+1}^{\prime}\left(k_{j, i}^{-}+k_{j, i}^{+}\right)+\sigma_{i+1, i+1}^{\prime} k_{j, i}^{+}\right] \\
& +\frac{130\left(1-S_{r}(1-H)\right)\left(n_{0}-\varepsilon_{j, i}\right)}{\left(\sigma_{1}^{\prime}-\sigma_{i, i}^{\prime}+130\right)^{2}}\left(\sigma_{j, 1+1}^{\prime}-\sigma_{j, i}^{\prime}\right) \text {. }
\end{aligned}
$$

\subsection{Les conditions aux limites}

Au moment $t=0$ du chargement, les surpressions interstitielles sont nulles dans le sol car aucune variation de volume de la phase liquide compressible ne s'est produite;

quel que soit i $\quad \sigma_{j, 1}^{\prime}=\sigma_{1}=$ const.

Sur la surface drainante, elles restent nulles tout au long de la consolidation;

quel que soit $i \quad \sigma_{1, i}^{\prime}=\sigma_{1}=$ const.

Le gradient hydraulique au contact du substratum imperméable est nul, puisque aucun flux de liquide ne le traverse. La fonction $\sigma^{\prime}(z)$ qui définit les isochrones de surpressions interstitielles, a une dérivée nulle pour $z=0$.

Une solution fictive $\sigma_{n+1,1+1}^{\prime}$, est calculée à chaque itération dans le plan situé immédiatement sous le substratum, avec la condition :

$$
\sigma_{n-1, i+1}^{\prime}=\sigma_{n+1, i+1}^{\prime} \text {. }
$$

La fonction $\sigma^{\prime}(z)$ est paire au voisinage du substratum; sa dérivée est continue, par conséquent $\frac{\partial \sigma^{\prime}(z)}{\partial z}=0$.

\section{Résultats des calculs et comparaison avec les mesures effectuées}

Les mesures sont relatives au remblai expérimental B de Cubzac-les-Ponts et à une série d'essais œdométriques réalisés sur l'argile intacte du même site (les 
essais ont été décrits antérieurement par Félix, 1980 c). Le comportement du sol est caractérisé dans les calculs, par un petit nombre de paramètres qui se mesurent facilement.

$\alpha, \beta$ et $\varepsilon_{f}$ de la fonction mesure de fluage, sont déterminés à partir des tassements de consolidation secondaire.

$a_{1}, b_{1}$ et $a_{2}, b_{2}$ de la fonction de contrainte sont déduits de la contrainte de préconsolidation des échantillons soit $\sigma_{\mathrm{p}}^{t}=35 \mathrm{kPa}$, et des résultats de deux essais de compression à long terme.

L'indice des vides initial $e_{0}$ et le degré de saturation $S_{\text {, }}$ dépendent des mesures pondérales effectuées sur chaque échantillon.

Enfin $\mathrm{A}$ et $\mathrm{B}$ qui lient l'indice des vides à la perméabilité par la relation (12), sont connus grâce à la série d'essais de perméabilité effectués sur le sol de Cubzacles-Ponts :

$$
A=0,88 \text { et } B=0,95 \text {. }
$$

Le programme Hydroflu simule donc la consolidation de monocouches de sol parfaitement individualisés, dont les tassements réels sont comparés aux résultats du calcul.

Les essais ont été conçus de façon à étudier l'influence de la grandeur de la charge appliquée et celle de la longueur de drainage. Quatre échantillons dont le degré de saturation moyen était égal à $92 \%$, ont été soumis à des compressions œdométriques d'une durée de 20 mois, sous des charges constantes égales à $40-80-160$ et $320 \mathrm{kPa}$.

Quatre autres échantillons, ayant un degré de saturation moyen égal à $96 \%$, et dont les longueurs de drainage étaient respectivement $5-10-20$ et $40 \mathrm{~mm}$ ont été chargés en quatre paliers identiques de $40 \mathrm{kPa}$ à intervalles de une semaine.

\subsection{Influence de la charge appliquée}

3.1.1 Sur les déformations dont les valeurs moyennes sont calculées par la formule (2) à partir de l'ensemble des déformations en chaque point du monocouche, obtenues par résolution numérique des équations de la consolidation. Sur la figure 2, elles sont représentées en fonction du logarithme du temps par une ligne continue; les points entourés de divers figurés géométriques correspondant aux déformations mesurées. Les traits pointillés représentent le fluage du squelette intergranulaire en l'absence de toute surpression interstitielle.

La simulation indique que la fraction de la déformation finale atteinte à la fin de la consolidation hydrodynamique diminue avec la charge appliquée. Plus celle-ci est petite, plus l'écart entre la courbe de déformation moyenne et celle du fluage du squelette s'amenuise. A la limite, la phase hydrodynamique tend à disparaitre et l'ensemble de la consolidation est régi par le fluage du squelette. Le coude de la courbe tassement-temps dans le diagramme semi-logarithmique, correspondant au temps $t_{90}$ et qui permet de déterminer le coefficient de consolidation $c_{v}$, n'apparaît pas pour la charge la plus petite; soit $40 \mathrm{kPa}$. Dans ce cas, l'évolution des tassements est très différente des prévisions déduites de la théorie de K. Terzaghi.

Par contre, quelle que soit la charge, la théorie de la consolidation proposée permet une interprétation correcte, tant de la durée de la phase hydrodynamique que des déformations moyennes. II y a convergence entre ces dernières, et le fluage du squelette sous la charge extérieure appliquée qui régit la consolidation secondaire du sol.

Fig. 2 Déformations calculées (courbes) et mesurées (points), et fluage virtuel du squelette en fonction du logarithme du temps

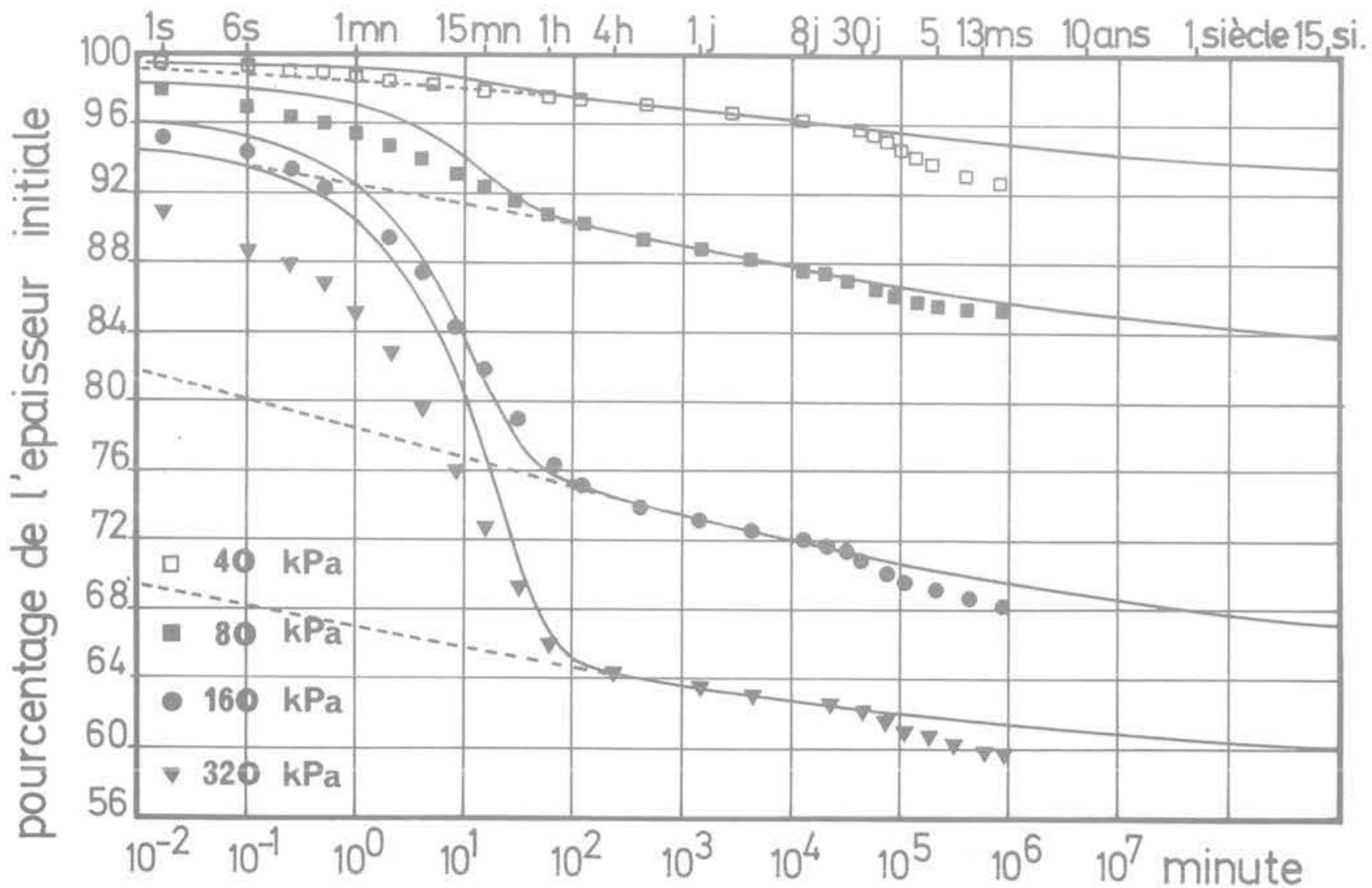




\subsubsection{Répercussion de la charge appliquée sur les surpressions interstitielles}

La consolidation hydrodynamique d'un sol imparfaitement saturé, dont le squelette flue, évolue différemment suivant qu'il est faiblement ou fortement surconsolidé.

Sur la figure 3 , sont tracées les courbes isochrones relatives à la consolidation, sous $320 \mathrm{kPa}$ d'un échantillon œdométrique du sol intact de Cubzac-lesPonts. Elles représentent, pour des valeurs du temps doubles les unes des autres, les surpressions interstitielles calculées par le programme Hydroflu, en fonction de la distance initiale au substratum imperméable. La contrainte avant chargement étant égale à $5 \mathrm{kPa}$ et la contrainte de préconsolidation à $35 \mathrm{kPa}$, le sol est faiblement surconsolidé.

Dans un premier temps, l'écoulement du fluide interstitiel n'exerce aucune influence sur la déformation. La consolidation se réduit à une compression non drainée; la pression du liquide interstitiel augmente tandis que son volume diminue.

La figure 5 représente la variation en fonction du logarithme du temps, du quotient de la surpression interstitielle la plus grande, calculée au contact du substratum imperméable, par la charge appliquée soit $320,160,80$ et $40 \mathrm{kPa}$ pour chacun des quatre échantillons œdométriques. La valeur maximale est atteinte presque instantanément, lorsque la charge appliquée est grande (160 et $320 \mathrm{kPa}$ ), et seulement au bout de 2 minutes pour $80 \mathrm{kPa}$, à cause de la viscosité plus grande du squelette sous de telles charges. Pour ces trois essais, elle est comparable à la différence entre la charge appliquée $\sigma_{1}$ et la contrainte de préconsolidation $\sigma_{\mathrm{p}}^{\prime}$ (voir tableau 1).

\begin{tabular}{c|c|c}
\hline $\begin{array}{c}\text { charge } \\
\text { appliquée } \\
\sigma_{1}\end{array}$ & $\begin{array}{c}\text { différence avec } \\
\text { la contrainte de } \\
\text { préconsolidation } \\
\sigma_{1}-\sigma_{1}^{\prime}\end{array}$ & $\begin{array}{c}\text { pression } \\
\text { interstitielle } \\
\text { maximale } \\
U_{\max }\end{array}$ \\
\hline $320 \mathrm{kPa}$ & $285 \mathrm{kPa}$ & $277 \mathrm{kPa}$ \\
$160 \mathrm{kPa}$ & $125 \mathrm{kPa}$ & $120 \mathrm{kPa}$ \\
$80 \mathrm{kPa}$ & $45 \mathrm{kPa}$ & $44 \mathrm{kPa}$ \\
\hline
\end{tabular}

Tableau 1

De ce point de vue, la simulation par le programme Hydroflu concorde avec les conclusions tirées par Abelev (1964) ou par Tavenas et al (1978) de l'étude des argiles intactes faiblement surconsolidées : les contraintes effectives sont, pendant toute la durée de la consolidation, supérieures à la contrainte $\sigma_{p}^{\prime}$ de préconsolidation.

Dans un deuxième temps, la déformation de l'ensemble du monocouche de sol est affecté par le drainage du liquide interstitiel. Les isochrones s'arrondissent et la valeur maximale de la surpression interstitielle décroît vers zéro. La diminution de la perméabilité au contact de la surface drainante, qui est due aux grandes déformations du sol, explique la prolongation de la consolidation sous les plus fortes charges.

Fig. 3 Isochrones caractéristiques de la consolidation de l'argile de Cubzac-les-Ponts sous une forte charge : $320 \mathrm{kPa}$
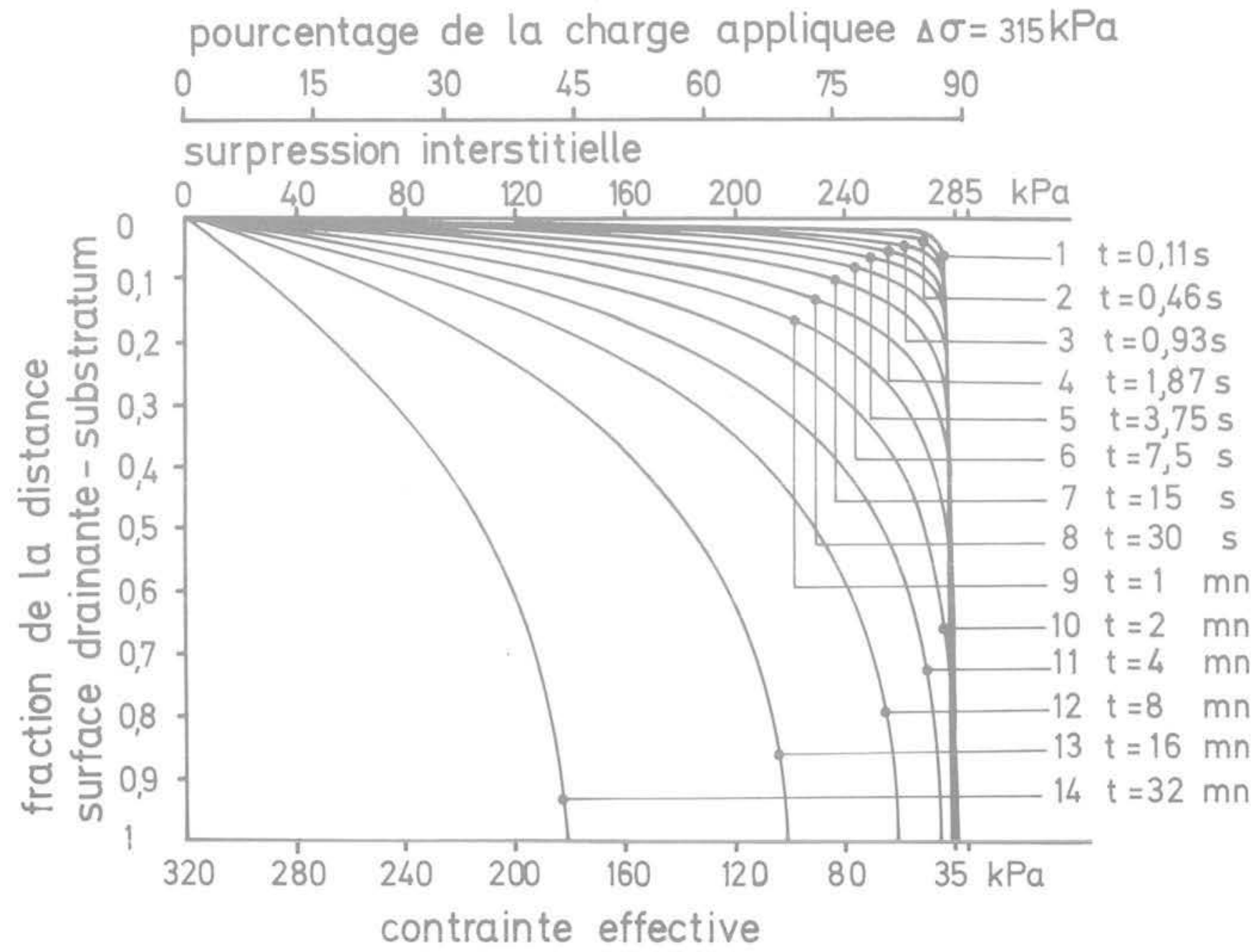
La figure 4 représente les courbes isochrones caractéristiques de la consolidation sous $40 \mathrm{kPa}$, d'un échantillon d'argile de Cubzac-les-Ponts qui est donc surconsolidé puisque $\sigma_{\mathrm{p}}^{\prime}=35 \mathrm{kPa}$.

Le fluage du squelette intergranulaire sous les faibles charges est lent, par conséquent la compression initiale en l'absence de drainage l'est aussi. Elle est accompagnée d'une croissance très sensible des surpressions interstitielles qui atteignent après une minute environ, une faible fraction de la charge appliquée; soit 35 pour cent. Les contraintes effectives restent toutefois nettement inférieures à la contrainte de préconsolidation; le comportement du sol est surconsolidé.

A la fin de la phase hydrodynamique de la consolidation, forsque la dissipation des surpressions interstitielles est suffisante, le passage dans le domaine normalement consolidé est marqué par une brusque diminution de la vitesse de dissipation. Sur la figure 5 , on observe une rupture de pente sur la courbe correspondante.

La diminution de la vitesse de dissipation contrebalance en quelque sorte l'augmentation de la compressibilité du sol.
Ainsi le passage des contraintes effectives au-delà de la contrainte de préconsolidation ne se traduit par aucune variation de la vitesse de déformation du sol, comme on peut le remarquer sur la figure 2.

3.2 L'influence de la distance de drainage a été étudiée pour quatre valeurs différentes du rapport $\frac{\sigma_{1}}{\sigma_{0}}$ de la contrainte totale appliquée au cours de la consolidation, sur celle appliquée préalablement. Après reconsolidation sous la contrainte $\sigma_{p}^{\prime}$ de préconsolidation, les échantillons dont la longueur de drainage est égale à $5-10-20$ et $40 \mathrm{~mm}$, ont été soumis à quatre paliers de chargement de $40 \mathrm{kPa}$ d'une durée de une semaine chacun.

\subsubsection{Influence sur les déformations}

Les déformations moyennes calculées par le programme Hydroflu, qui correspondent à la consolidation sous le premier palier de charge $\frac{\sigma_{1}}{\sigma_{0}}=2,15$, sont

Fig. 4 Isochrones caractéristiques de la consolidation de l'argile de Cubzac-les-Ponts sous une faible charge: $40 \mathrm{kPa}$ pourcentage de la charge appliquee $\Delta \sigma=35 \mathrm{kPa}$

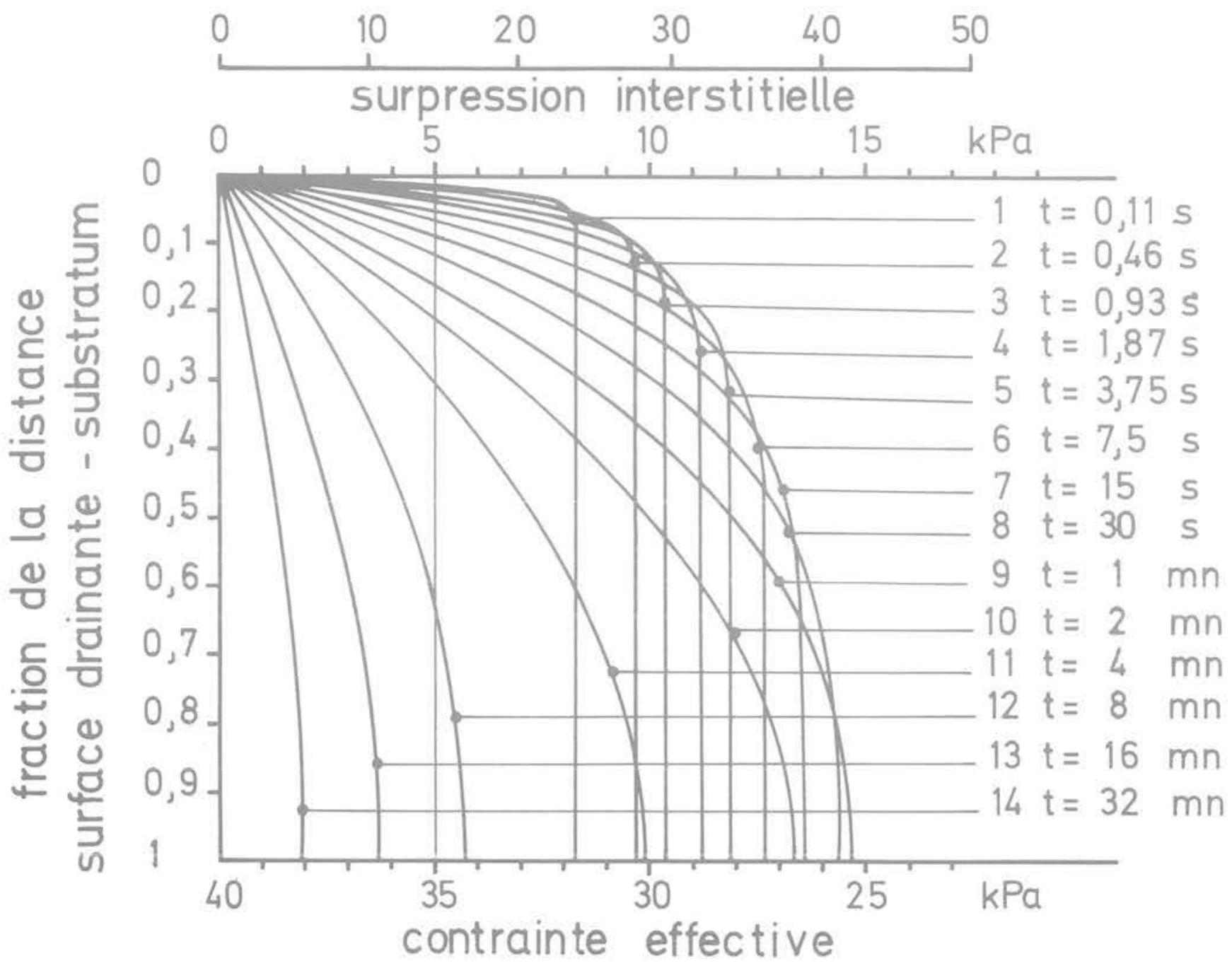




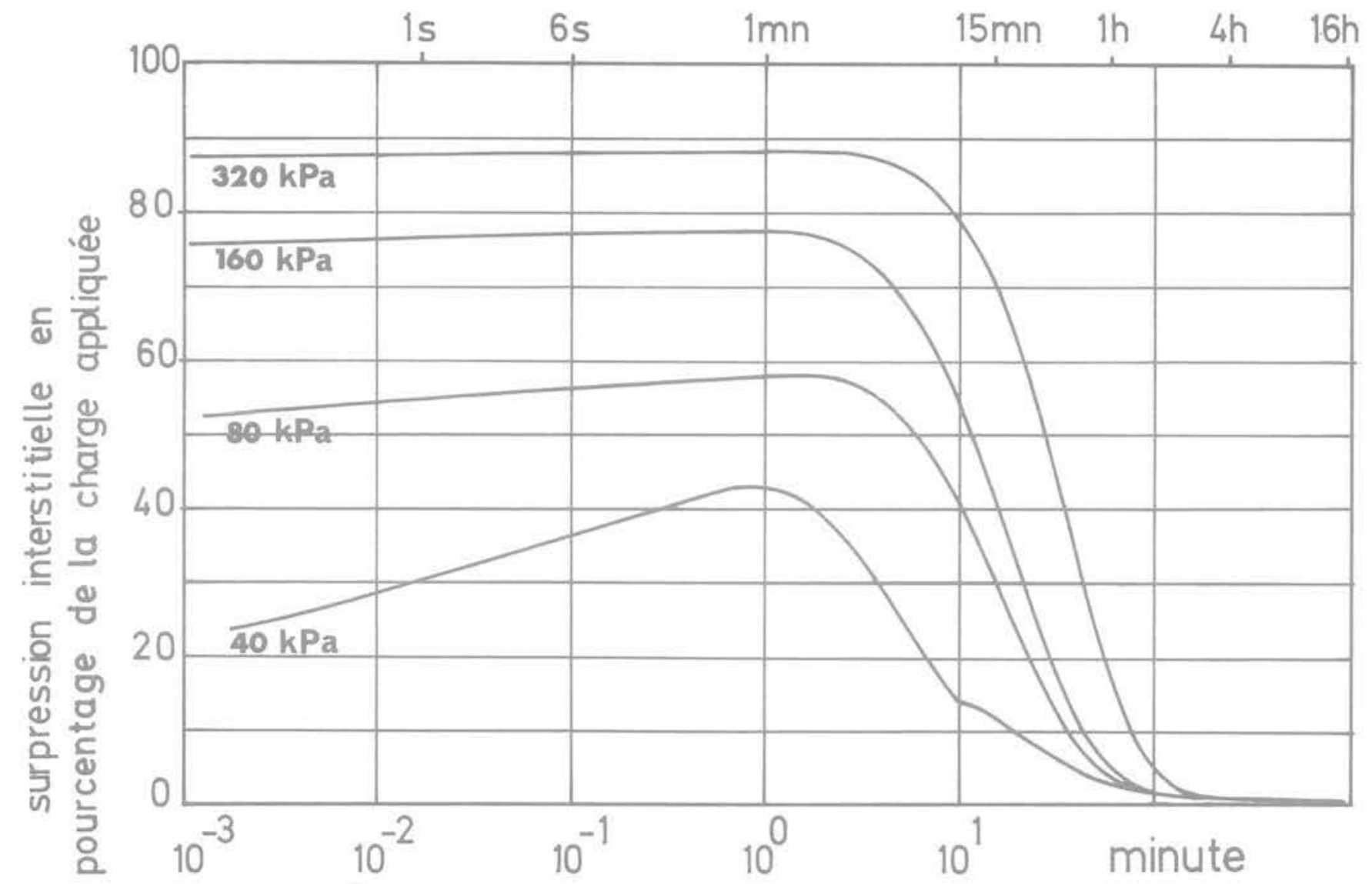

Fig. 5 Evolution dans le temps de la surpression interstitielle maximale rapportée à la charge appliquée

Fig. 6 Déformations calculées (courbes) et mesurées (points) en fonction du logarithme du temps de quatre échantillons reconsolidés sous $35 \mathrm{kPa}$ et soumis à une charge de $40 \mathrm{KPa}$;

les longueurs de drainage sont: $\nabla 40 \mathrm{~mm} \triangle 20 \mathrm{~mm}$ ㅁ $10 \mathrm{~mm} \cdot 5 \mathrm{~mm}$

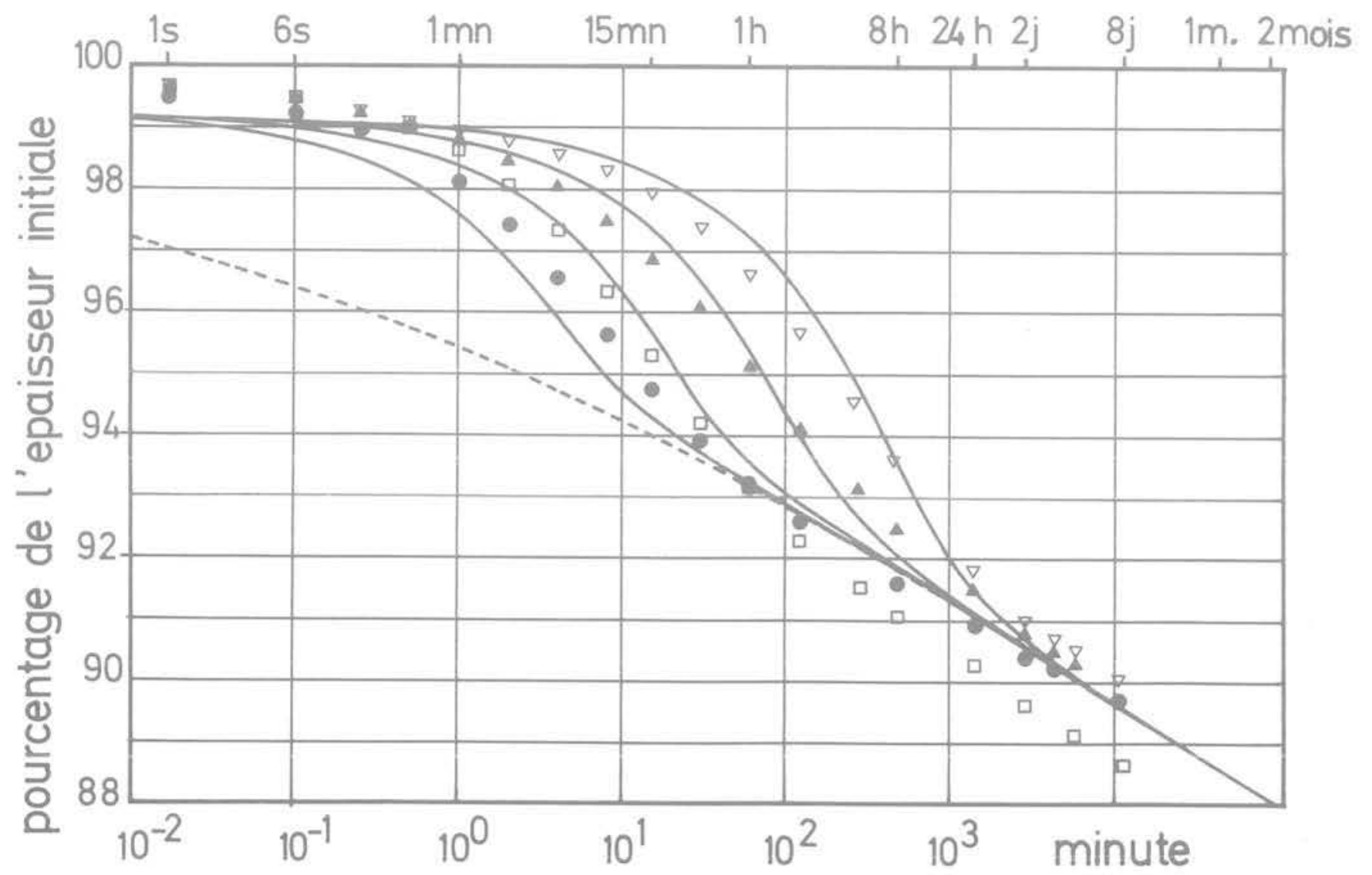


tracées sur la figure 6 en fonction du logarithme du temps, ainsi que les déformations mesurées et le fluage du squelette en l'absence de toute surpression interstitielle. La représentation est identique à celle de la figure 2. Les calculs restituent correctement les déformations mesurées au cours de l'ensemble de la consolidation, tant de la phase secondaire qui coïncide avec le fluage du squelette sous la charge extérieure, que de la phase hydrodynamique dont l'achèvement est marqué par un coude de la courbe tassementtemps. La construction de Casagrande permet de déterminer le temps $t_{90}$. En accord avec les prévisions de la théorie classique, la durée de la phase hydrodynamique est proportionnelle au carré de la longueur de drainage; pour une longueur double elle est approximativement multipliée par 4. Mais, en contradiction avec celles-ci, on observe que les déformations calculées ou mesurées après quelques secondes ne sont pas négligeables.

Elles sont dues au fluage du squelette et à la compression de la phase gazeuse, et ne dépendent pas de la longueur de drainage. D'après le calcul comme d'après l'expérience, la déformation des échantillons à la fin de la consolidation hydrodynamique diffère d'un échantillon à l'autre; le squelette étant visqueux, la déformation finale croît avec la durée de cette phase et donc avec la longueur de drainage.

Les résultats relatifs au quatrième palier de charge sont représentés d'une manière analogue sur la figure 7. La contrainte appliquée passe de la valeur $\sigma_{0}=195 \mathrm{kPa}$ à $\sigma_{1}=235 \mathrm{kPa}$, le rapport $\frac{\sigma_{1}}{\sigma_{0}}$ égalant 1,2 .
Les calculs, là encore, simulent correctement les résultats d'expérience bien que les déformations soient légèrement surestimées. Les déformations tant calculées que mesurées, sont proches de la courbe de fluage du squelette sous la charge extérieure. L'influence des surpressions interstitielles sur la consolidation est réduite, pour des taux d'accroissement de la charge extérieure qui sont aussi faibles. Elle existe cependant, puisque les déformations dépendent de la longueur de drainage. La dissipation complète des surpressions interstitielles est beaucoup plus graduelle que pour le premier palier de charge, et ne correspond à aucun coude de la courbe tassementtemps. Elle est également beaucoup plus longue, et n'intervient pour les échantillons les plus épais qu'après plusieurs jours.

\subsubsection{Influence de la longueur de drainage sur les surpressions interstitielles}

La figure 8 correspond au premier palier de charge de $40 \mathrm{kPa}$. Elle représente, en fonction du logarithme du temps, le quotient de la surpression interstitielle maximale calculée au contact du substratum imperméable et de l'incrément de charge extérieure.

Dans une première période, la longueur de drainage est sans influence. Au contact du substratum le fluide interstitiel est comprimé, mais ne s'écoule pas. La surpression atteint quasi-instantanément, des valeurs d'autant plus importantes que le degré de saturation est grand $(75 \%$ de la charge appliquée pour $\mathrm{S}_{\mathrm{r}}=96 \%$ ), puis subit une croissance légère, réglée par

Fig. 7 Déformations calculées (courbes) et mesurées (points) en fonction du logarithme du temps de quatre échantillons reconsolidés sous $195 \mathrm{kPa}$ et soumis à une charge de $40 \mathrm{KPa}$; les longueurs de drainage sont : $\nabla 40 \mathrm{~mm} \triangle 20 \mathrm{~mm}$ = $10 \mathrm{~mm} \quad 5 \mathrm{~mm}$

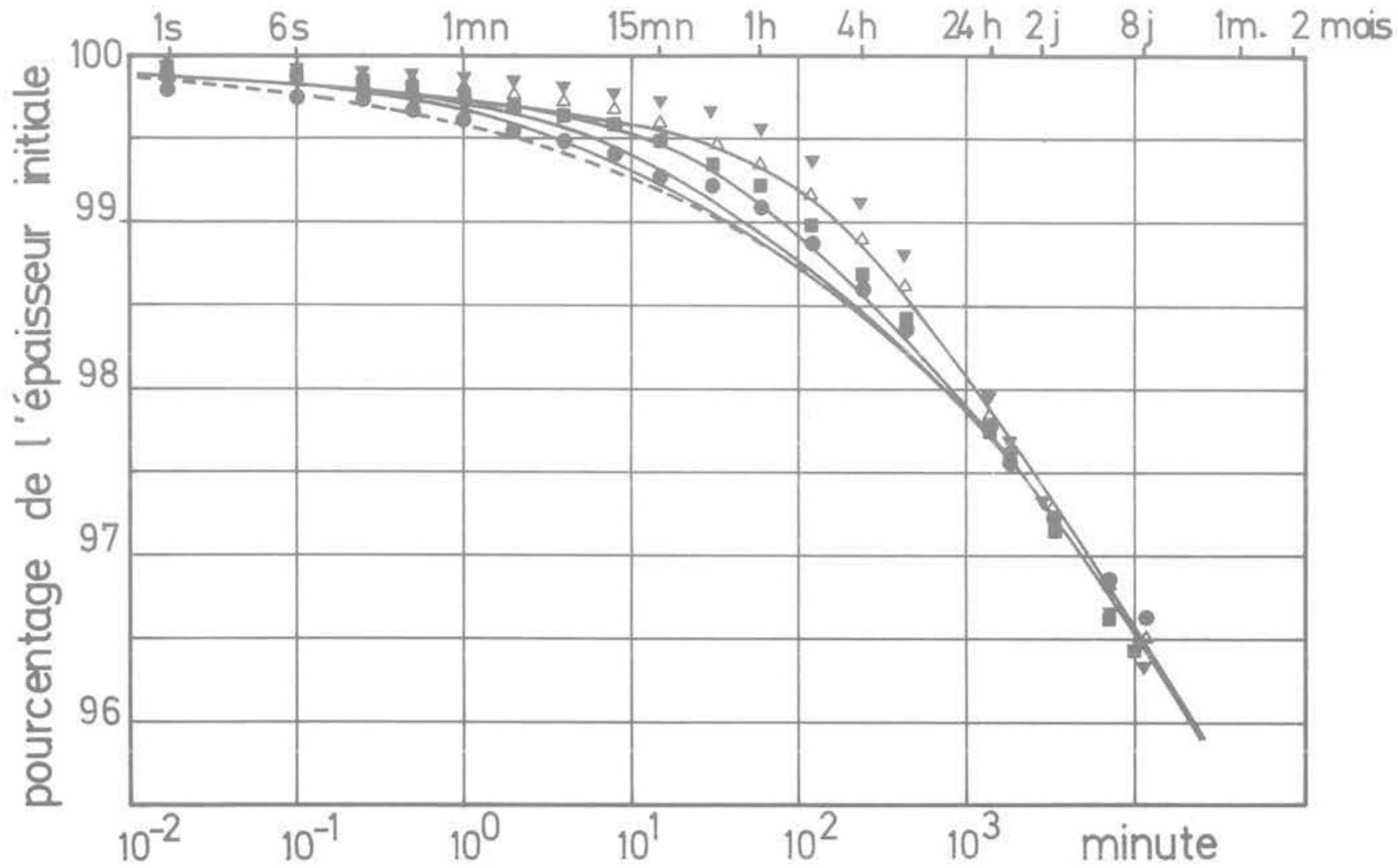


le fluage du squelette intergranulaire.

Pour les échantillons ayant les plus petites longueurs de drainage, l'intervention de la dissipation par écoulement est plus rapide, la surpression interstitielle au contact du substratum culmine au bout d'un temps plus court, et le maximum atteint représente une fraction moindre de la charge appliquée. Le tableau 2 récapitule pour les différentes longueurs de drainage, la valeur de ces maxima et le temps correspondant, qui est approximativement multiplié par 4 lorsque la longueur $h$ est doublée. On remarque que les surpressions interstitielles restent dans tous les cas inférieures à la charge appliquée.

\begin{tabular}{c|c|c}
\hline $\begin{array}{c}\text { longueur de } \\
\text { drainage }\end{array}$ & $\begin{array}{c}\text { répercussion } \\
\text { maximale de } \\
\text { la charge sur } \\
\text { la surpression } \\
\text { interstitielle }\end{array}$ & $\begin{array}{c}\text { temps au bout } \\
\text { duquel ce maximum } \\
\text { est atteint }\end{array}$ \\
\hline $5 \mathrm{~mm}$ & $85 \%$ & $25 \mathrm{~s}$ \\
$10 \mathrm{~mm}$ & $87 \%$ & $1 \mathrm{mn} 40 \mathrm{~s}$ \\
$20 \mathrm{~mm}$ & $89 \%$ & $7 \mathrm{mn}$ \\
$40 \mathrm{~mm}$ & $90 \%$ & $30 \mathrm{mn}$ \\
\hline
\end{tabular}

Tableau 2

La figure 9, qui présente les mêmes résultats relatifs au dernier des quatre paliers de charge de $40 \mathrm{kPa}$, souligne l'influence de la viscosité du squelette intergranulaire sur l'évolution des surpressions interstitielles. Un taux d'accroissement de la charge extérieure appliquée égale à 1,20 , ne peut provoquer qu'un fluage très lent du squelette. La compression initiale en l'absence de drainage se traduit par des surpressions tout d'abord négligeables, puis qui croissent sensiblement sur une période, fonction de la longueur de drainage. Les valeurs maximales atteintes lorsque la dissipation par écoulement commence, et le temps correspondant sont indiqués sur le tableau 3. Les valeurs décroissent fortement avec la longueur de drainage. Par rapport à celles obtenues pour le premier palier de charge, elles apparaissent comme plus petites, et le temps nécessaire pour les atteindre est presque deux fois plus long.

\begin{tabular}{c|c|c}
\hline $\begin{array}{c}\text { longueur de } \\
\text { drainage }\end{array}$ & $\begin{array}{c}\text { répercussion } \\
\text { maximale de } \\
\text { la charge sur } \\
\text { la surpression } \\
\text { interstitielle }\end{array}$ & $\begin{array}{c}\text { temps au bout } \\
\text { duquel ce maximum } \\
\text { est atteint }\end{array}$ \\
\hline $5 \mathrm{~mm}$ & $27 \%$ & $50 \mathrm{~s}$ \\
$10 \mathrm{~mm}$ & $35 \%$ & $3 \mathrm{mn}$ \\
$20 \mathrm{~mm}$ & $43 \%$ & $12 \mathrm{mn}$ \\
$40 \mathrm{~mm}$ & $53 \%$ & $50 \mathrm{mn}$ \\
\hline
\end{tabular}

Tableau 3

La décroissance régulière des surpressions interstitielles vers les valeurs nulles, qui marque la fin de la consolidation hydrodynamique, intervient légèrement plus tard pour le quatrième palier. Elle n'est pas complètement achevée après 6 jours, pour l'échantil-

Fig. 8 Evolution dans le temps des surpressions interstitielles maximales rapportées à la charge appliquée de quatre échantillons reconsolidés sous $35 \mathrm{kPa}$ et soumis à une charge de $40 \mathrm{kPa}$, pour des longueurs de drainage égales à : 40 - 20 - 10 $5 \mathrm{~mm}$

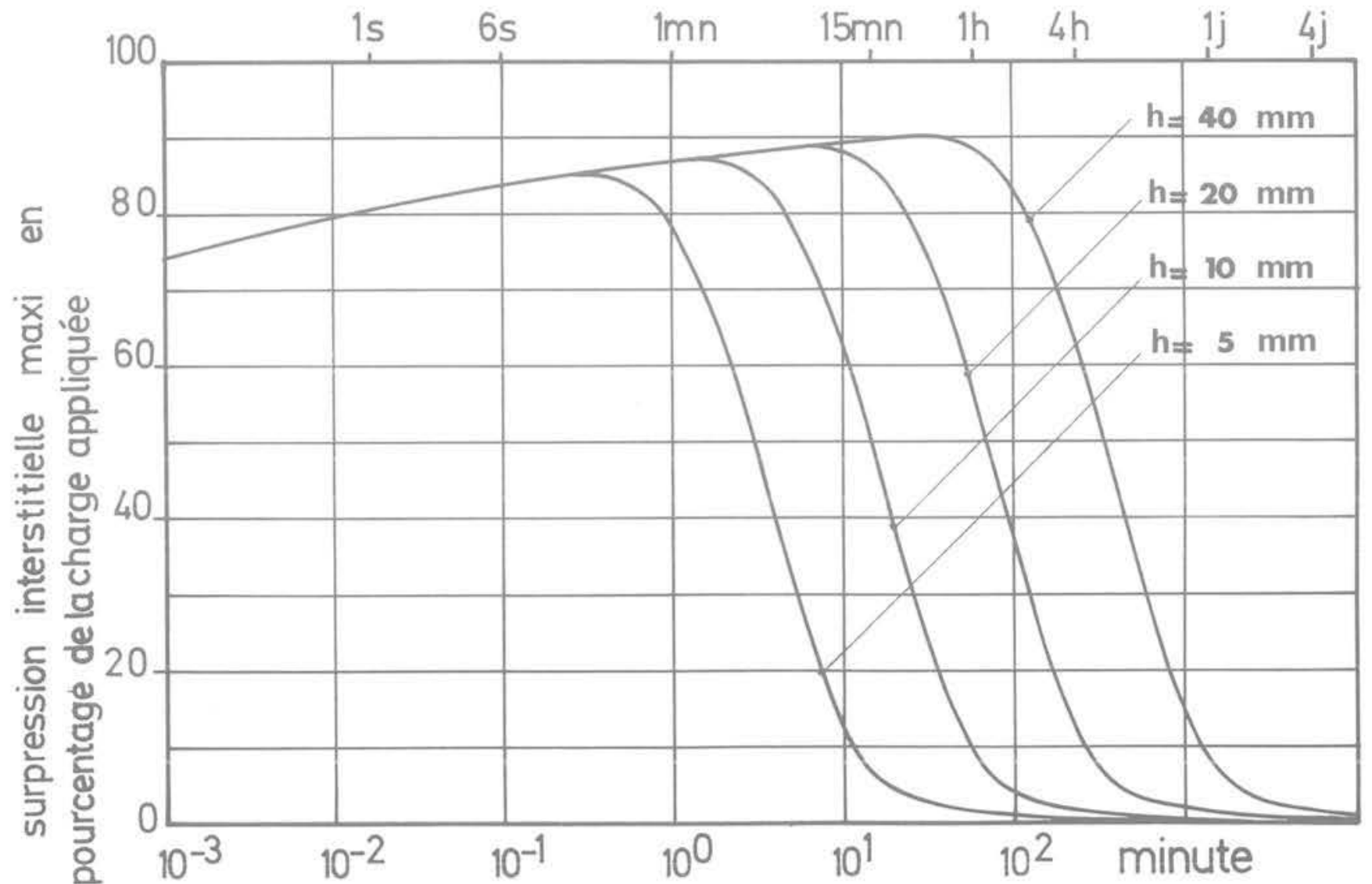


Ion le plus épais $(\mathrm{d}=40 \mathrm{~mm})$. En accord avec les résultats obtenus par Meschyan (1967), la simulation par le programme Hydroflu suggère que l'augmentation des surpressions interstitielles, et donc leur influence sur la déformation moyenne, sont d'autant plus faibles que le squelette intergranulaire est plus raide ou, ce qui revient au même, que le taux d'accroissement de la charge extérieure $\frac{\sigma_{1}}{\sigma_{0}}$ est plus petit. Mais le temps nécessaire pour atteindre la valeur maximale des surpressions interstitielles, ainsi que leur complète dissipation, est en général beaucoup plus long.

\subsection{Comparaison avec les mesures faites sous le remblai expérimental $B$ de ubzac-les-Ponts}

Ce remblai de $24 \mathrm{~m}$ de large est construit sur un horizon d'argile molle de $9 \mathrm{~m}$ d'épaisseur, qui repose sur un substratum drainant. Le toit de la nappe est situé en moyenne à $1 \mathrm{~m}$ sous le niveau du sol. Le remblai exerce une pression de $48 \mathrm{kPa}$.

La simulation de la déformation moyenne du sol de fondation et de la dissipation des surpressions interstitielles est basée sur une série d'approximations.

- La consolidation est supposée unidimensionnelle.

- Les contraintes totales avant et après construction, sont considérées comme identiques en tout point du sol, et égales à celles supportées par les quatre échantillons de différentes longueurs de drainage lors du premier palier de charge, bien que la grandeur de celui-ci: $40 \mathrm{kPa}$ soit nettement inférieure à la pression exercée par le remblai.

Les paramètres du calcul, et en particulier ceux du fluage du squelette, sont ceux des quatre échantillons (à l'exception bien sûr de la longueur de drainage).

Compte tenu de ces hypothèses, les calculs ont été effectués pour les trois valeurs suivantes du degré de saturation: $S_{r}=0,92-0,96$ et 1 . Sur la figure 10 , les déformations sont tracées en fonction du logarithme du temps pour une période allant de 1 seconde à 15 siècles. Les valeurs déterminées à partir des tassements mesurés sous le remblai, sont représentés par des carrés noirs. Les déformations calculées et mesurées relatives aux quatre échantillons œdométriques, y figurent également.

II apparaît tout d'abord que la compressibilité du fluide interstitiel influe sur les déformations tant que les surpressions interstitielles restent élevées; c'est-à-dire au début de la consolidation hydrodynamique, dont la durée totale d'après la simulation dépasse un siècle. A son achèvement, la déformation est égale à $15 \%$ et approche la déformation finale du squelette : $16,5 \%$, vers laquelle la fonction de fluage tend asymptotiquement. Dans un tel cas, on peut donc conclure que le fluage du squelette n'intervient pratiquement que pendant la consolidation primaire.

Fig. 9 Evolution dans le temps des surpressions interstitielles maximales rapportées à la charge appliquée de quatre échantillons reconsolidés sous $195 \mathrm{kPa}$ et soumis à une charge de $40 \mathrm{kPa}$, pour quatre longueurs de drainage

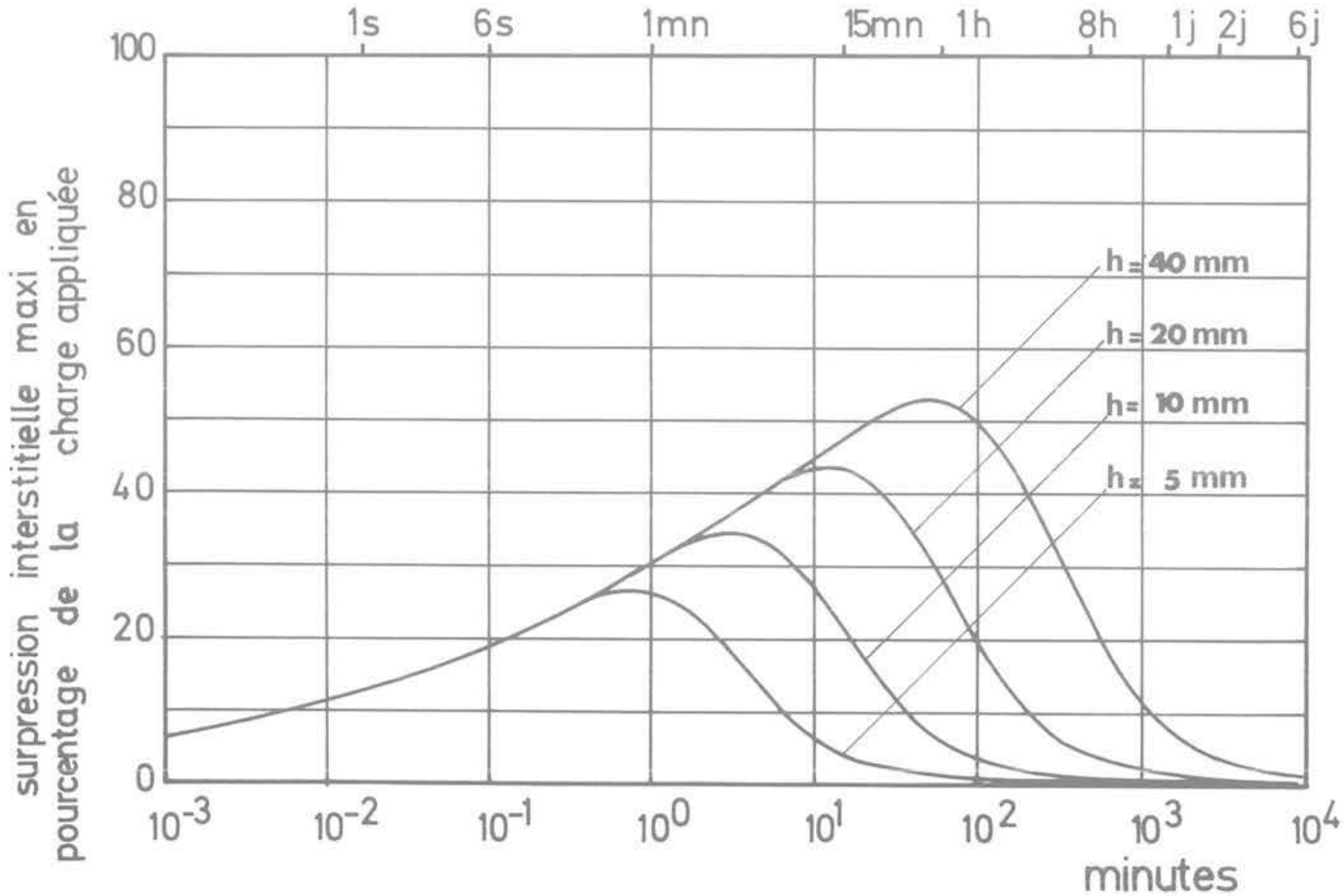




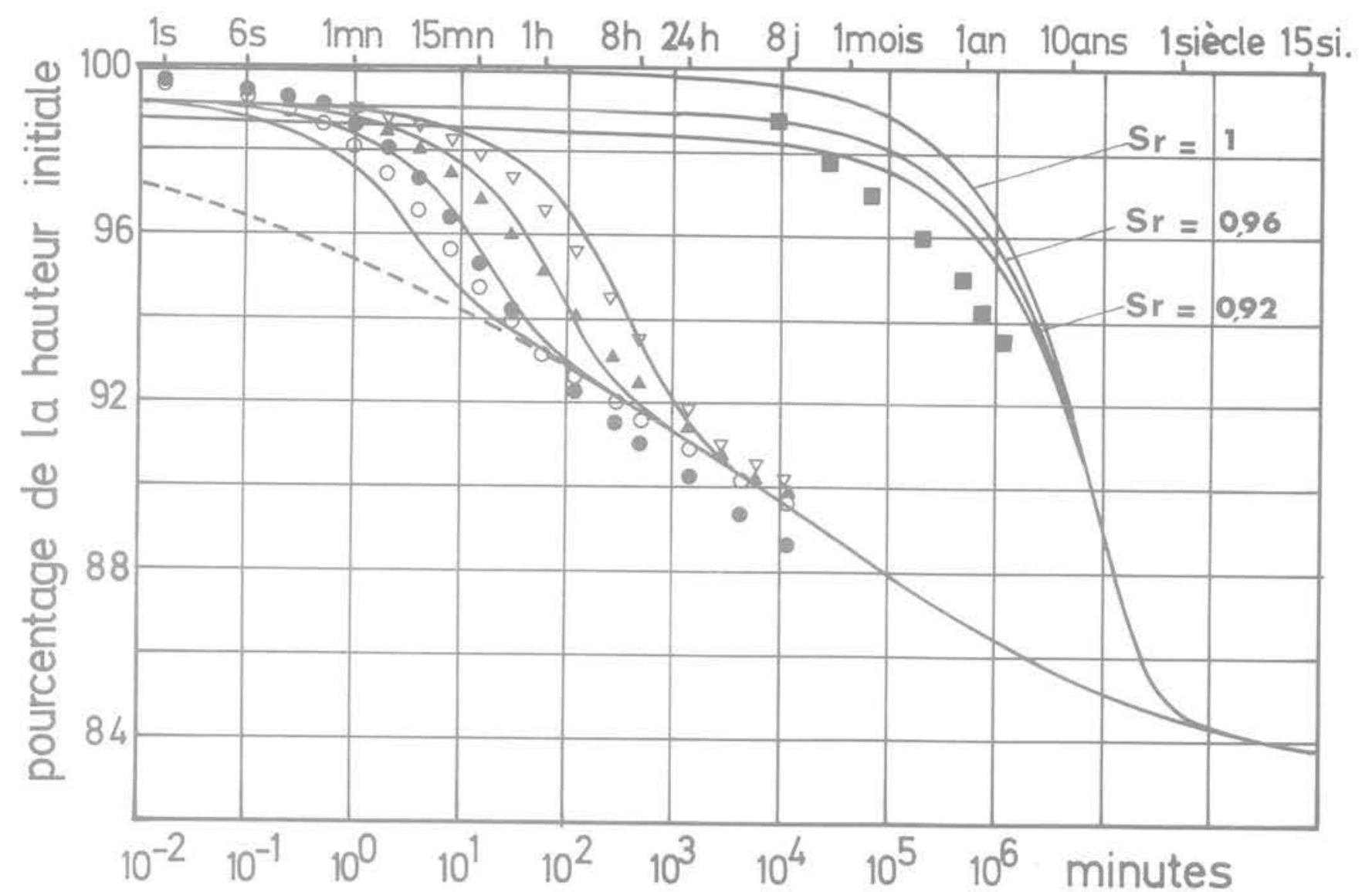

Fig. 10 Déformations comparées de quatre échantillons (longueur de drainage : $40 \cdot 20-10 \cdot 5 \mathrm{~mm}$ ) et du sol de fondation du remblai $B$ de Cubzac-les-Ponts $(h=4,5 \mathrm{~m})$ pour 3 degrés de saturation $(0,92$ - 0,96 - 1)

\section{Deuxième partie : Abaques en variables réduites de la consolidation et du fluage}

\section{Formulation simplifiée des équations de la consolidation}

La théorie de la consolidation formulée dans la première partie, montre que le fluage du squelette intergranulaire et la compressibilité apparente du fluide interstitiel permettent d'expliquer l'évolution réelle des tassements et des surpressions interstitielles. Elle constitue donc un progrès par rapport à la théorie classique de K. Terzaghi. Cependant son utilisation pratique ne peut être que limitée, puisque la résolution de ses équations est numérique et nécessite l'emploi du programme de calcul Hydroflu, dont la diffusion malgré sa simplicité et son faible coût d'utilisation, sera toujours restreinte.

Pour disposer de solutions directement accessibles, sous forme d'abaques ou de tableaux, il faut simplifier la théorie précédente et donc abandonner un certain nombre d'hypothèses.

Les résultats décrits dans la première partie, de même que certaines recherches récentes, permettent de juger de l'impact des différentes hypothèses, sur les résultats.
- L'hypothèse des grandes déformations n'est intéressante que si celles-ci dépassent $15 \%$. L'erreur que son abandon introduit est, en tout cas, inférieure au pourcentage que représente la déformation maximale.

- La compressibilité du fluide interstitiel a une grande influence sur l'évolution des surpressions et des tassements au début de la consolidation hydrodynamique, qui s'estompe par la suite.

- L'évolution du module cedométrique du sol et de sa perméabilité avec l'indice des vides, ont des effets antagoniques. Si l'un est supposé constant, il est logique de supposer que l'autre l'est aussi. Mesri et Rokhsar (1974) ont montré que l'effet de leur variation conjointe pouvait être nul, si l'indice de compression $C_{c}$ était égal au coefficient $A$ de la relation (12). Dans ce cas, l'hypothèse de la constance est parfaitement acceptable.

Le coefficient de perméabilité est constant, et simultanément la fonction de contrainte est linéarisée. Elle s'identifie à l'accroissement de contrainte effective $F\left(\sigma_{j}^{\prime}\right)=\Delta \sigma^{\prime}=\sigma^{\prime}-\sigma_{0}$. 
- Les hypothèses relatives à l'équation d'état du squelette intergranulaire s'avèrent donc, par élimination, être déterminantes. Dans la formation simplifiée, cette équation est celle de la théorie du fluage hérédiatire linéaire :

$$
\varepsilon(t)=\int_{0}^{t} K(t-\tau) \Delta \sigma^{\prime}(\tau) d \tau
$$

La fonction d'influence $K(t)$ est définie par sa primitive

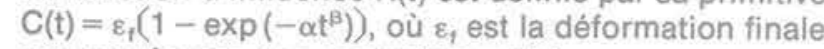
provoquée par une contrainte unité.

La déformation finale à la fin de la consolidation, est donc égale à :

$$
\Delta \sigma_{1}^{\prime} \varepsilon_{\mathrm{f}}=\left(\sigma_{1}^{\prime}-\sigma_{0}^{\prime}\right) \varepsilon_{1}=\varepsilon_{\infty}
$$

et le module œdométrique à long terme, à :

$$
E_{\mathrm{fl}}=\frac{1}{\varepsilon_{\mathrm{f}}}=\frac{\Delta \sigma_{1}^{\prime}}{\varepsilon_{\infty}}
$$

L'équation de la théorie de la consolidation simplifiée s'écrit finalement :

$$
\frac{\varepsilon(z, t)}{\partial t}=\frac{\partial}{\partial t}\left[\int_{0}^{t} K(t-\tau) \Delta \sigma^{\prime}(z, \tau) d \tau\right]=\frac{k}{\gamma_{w}} \frac{\partial^{2} \Delta \sigma^{\prime}(z, t)}{\partial z^{2}} .
$$

2 Les variables réduites sont définies de la manière suivante :

accroissement de contrainte effective réduit :

$$
\chi=\frac{\sigma^{\prime}-\sigma_{0}}{\sigma_{1}^{\prime}-\sigma_{0}}=\frac{\Delta \sigma^{\prime}}{\Delta \sigma_{1}^{\prime}},
$$

déformation réduite :

$$
\eta=\frac{\varepsilon}{\Delta \sigma_{1}^{\prime} \varepsilon_{f}}=\frac{\varepsilon}{\varepsilon_{\infty}},
$$

mesure de fluage réduite: $\quad c(t)=1-\exp \left(-\alpha t^{\beta}\right)$.

Toutes ces variables sont sans dimension.

La formulation implicite des équations (24) en différences finies, s'écrit au temps $t_{i}$ et dans le plan de cote $z_{j}$ :

$$
\begin{aligned}
& \eta_{j, i+1}-\eta_{j, i}=x_{j, i}\left(c\left(t_{i+1}\right)-c\left(t_{i}\right)\right)+\sum_{k=1}^{i-1}\left(x_{j, k+1}-x_{j, k}\right) \\
& \left(c\left(t_{i+1}-t_{k}\right)-c\left(t_{i}-t_{k}\right)\right)+\left(x_{j, i+1}-x_{j, i}\right) c\left(t_{i+1}-t_{j}\right) \\
& \eta_{j, i+1}-\eta_{j, 1}=\frac{n^{2}}{2 h^{2}} \frac{k E_{f 1}}{\gamma_{w}}\left(t_{i+1}-t_{i}\right)\left(x_{j-1, i}-2 x_{j, i}+x_{j+1, i}\right. \\
& \left.\quad+x_{j-1, i+1}-2 x_{j, i+1}+x_{j+1, i+1}\right), \quad(25)
\end{aligned}
$$

où le monocouche d'épaisseur $h$ est divisé en $n$ segments égaux.

Dans les équations (25), intervient le coefficient de consolidation fluage $c_{v t}=\frac{k E_{f l}}{\gamma_{w}}$ qui ne diffère du coefficient $c_{v}$ de la théorie de $\mathrm{K}$. Terzaghi que par la définition du module codométrique. Dans le mode opératoire, il lie un accroissement de contrainte effective à une déformation supposée élastique instantanée du squelette, qui est mesurée après 24 heures de consolidation à l'œdomètre, tandis que $\mathrm{E}_{n}$ lie cet accroissement, à une déformation différée atteinte au bout d'un temps infini.

On définit donc un "temps réduit", analogue au facteur temps de la théorie de $K$. Terzaghi : $T=\frac{t c_{v t}}{h^{2}}$. Ce qui entraîne la modification suivante de la mesure de fluage réduite :

$$
\bar{c}(T)=c(t)=1-\exp \left(-\overline{\alpha T^{\beta}}\right)=1-\exp \left(-\alpha t^{\beta}\right)
$$

par conséquent $\quad \bar{\alpha}=\alpha\left(\frac{h^{2}}{c_{v t}}\right)^{\beta}$.

Les équations (25) dans lesquelles $T$ est substitué à t et $\bar{c}(T)$ à $c(t)$, ne contiennent plus que deux paramètres $\beta$ et $\bar{\alpha}$, qui sont tous deux issus des caractéristiques de la mesure de fluage $\mathrm{C}(\mathrm{t})$, le deuxième étant fonction d'un coefficient d'échelle égal à $\frac{h^{2}}{c_{v f}}$.

La résolution de ces équations permet de calculer en fonction du temps réduit $T$, le degré de déformation moyen du monocouche égal à la moyenne des déformations réduites $\eta_{j, 1}$ et son degré de consolidation défini comme la moyenne des accroissements de contrainte effective réduits $\chi_{\mathrm{j}, \mathrm{j}}$.

Ils sont représentés sous la forme d'une série d'abaques, caractérisés par différentes valeurs de $\beta$, chacun renfermant une série de courbes qui correspondent aux valeurs de $\bar{\alpha}$ (voir les figures 11 et 12).

Une série de 13 abaques, du degré de consolidation et du degré de déformation a déjà été publiée (Félix, $1980 \mathrm{~b})$. Les valeurs de $\beta$ et $\bar{\alpha}$ retenues, sont suffisamment variées pour couvrir l'ensemble des problèmes possibles.

3 Conclusions théoriques auxquelles conduit le déchiffrage des abaques.

Les courbes du degré de consolidation et du degré de déformation qui portent le numéro 1 , coïncident entre elles et avec la solution classique de $\mathrm{K}$. Terzaghi, quelle que soit la valeur de $\beta$.

Les courbes correspondent aux plus grandes valeurs de $\bar{\alpha}$ pour lesquelles la fonction $\bar{c}(T)$ peut être assimilée à une fonction échelon de Heaviside $h(T)$, qui est nulle lorsque $T$ est nul, et égale à 1 pour les valeurs positives de T. Cette fonction décrit la déformation réduite, entièrement instantanée, d'un ressort linéaire qui simule le comportement du squelette dans la théorie classique. Si l'on remplace dans les équations (25) $\bar{c}(T)$ par $h(T)$; la première équation donne $\eta=\chi$, et par conséquent la deuxième se réduit à la formulation en différences finies, de l'équation de la consolidation de la théorie classique:

$$
\frac{\partial U}{\partial t}=\frac{C_{v}}{h^{2}} \frac{\partial^{2} U}{\partial z^{2}}
$$

Le paramètre $\bar{\alpha}$ augmente avec $\alpha$ et avec le coefficient d'échelle $\frac{h^{2}}{c_{v t}}$ porté à la puissance $\beta$.

Les grandes valeurs de $\alpha$ impliquent une croissance rapide de la mesure de fluage vers la déformation finale, qui n'est obtenue expérimentalement que par application de fortes charges sur des matériaux compressibles.

Ceci signifie, que l'évolution de la consolidation réelle est d'autant plus conforme aux prévisions de la théorie classique, que l'épaisseur du monocouche est grande; en d'autres termes le fluage du squelette intergranulaire exerce une influence beaucoup plus grande sur la consolidation d'un échantillon œedométrique, que sur celle d'un sol de fondation. De même, cette conformité augmente pour les sols de faible perméabilité (k petit), ou très compressibles; c'est-à-dire qui sont dotés d'un module $E_{n}$ petit, et surtout d'un coefficient rhéologique $\alpha$ qui est grand.

La théorie de K. Terzaghi constitue donc un cas particulier limite de la théorie proposée. Toutefois, son 
Abaque du degré de consolidation

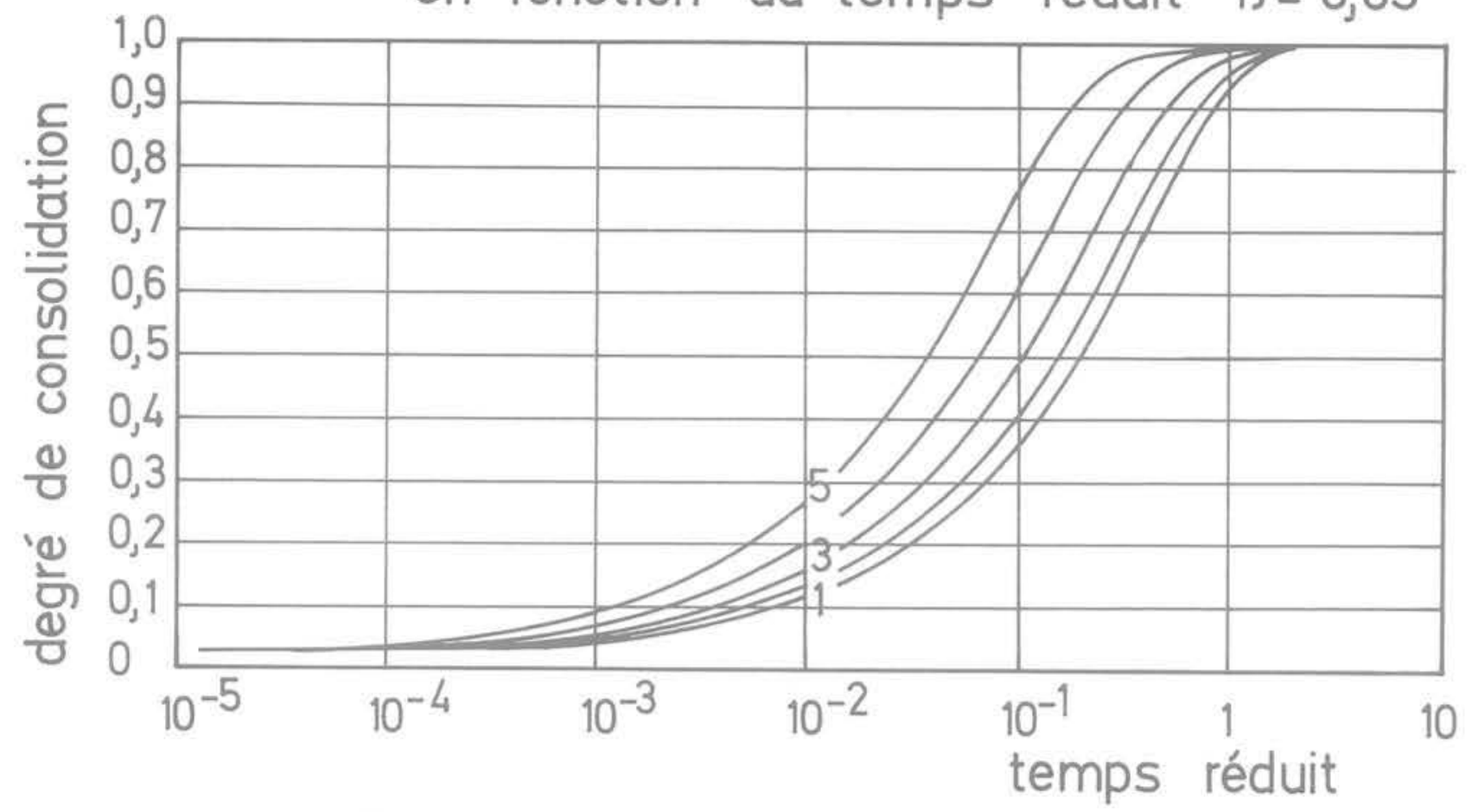

en fonction du temps réduit $\beta=0,05$

courbe $1 \quad \bar{\alpha}=6,0 \quad$ courbe $3 \quad \bar{\alpha}=0,89$ courbe $5 \quad \bar{\alpha}=0,25$

$2 \bar{\alpha}=1,7 \quad 4 \quad \bar{\alpha}=0,47$

Abaque du degré de tassement

en fonction du temps réduit $\beta=0,05$

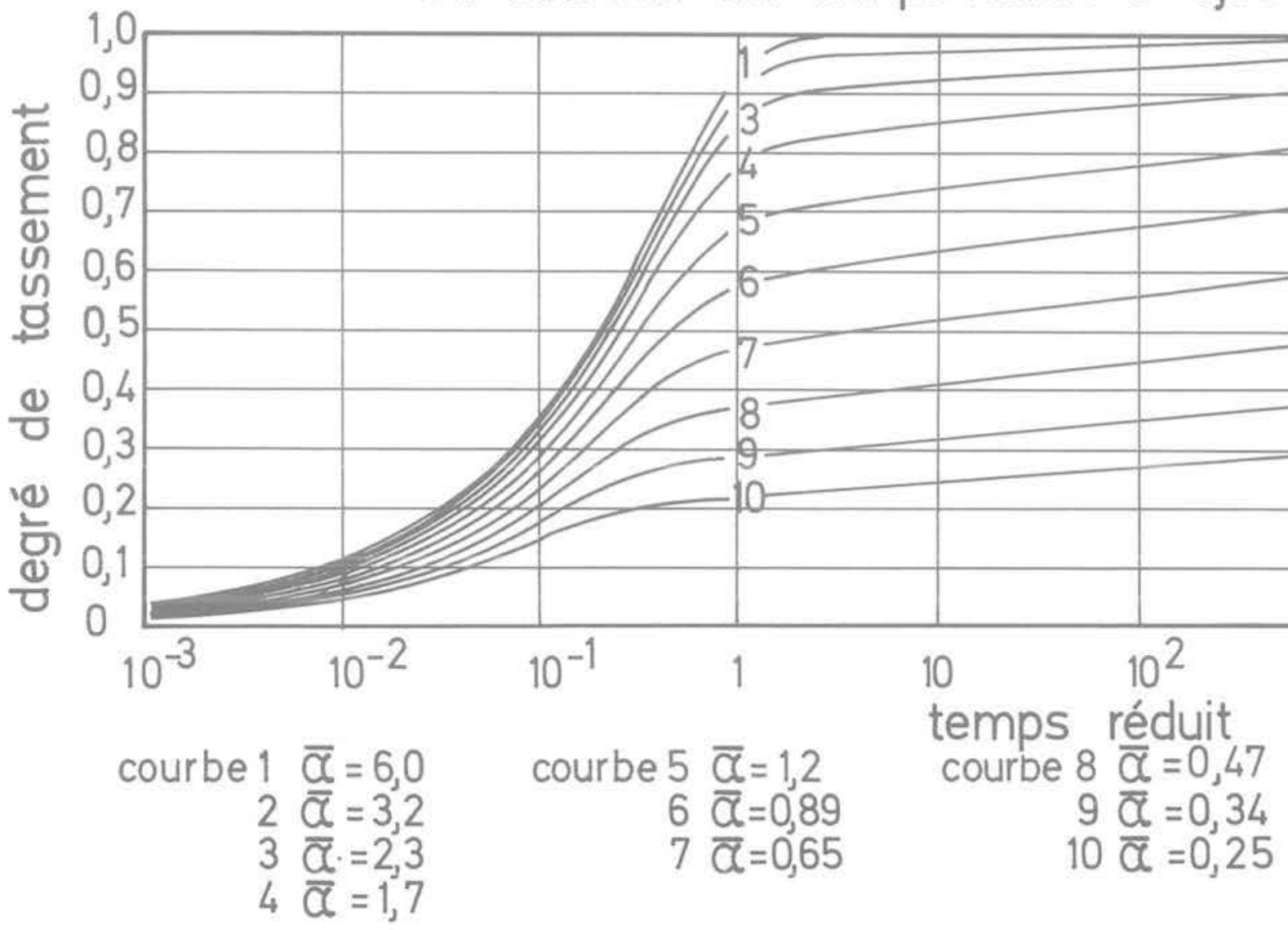

Fig. 11 Abaques des degrés de consolidation et de tassement de sols dont la viscosité est petite $(\beta=0,05)$ 
Abaque du degré de consolidation

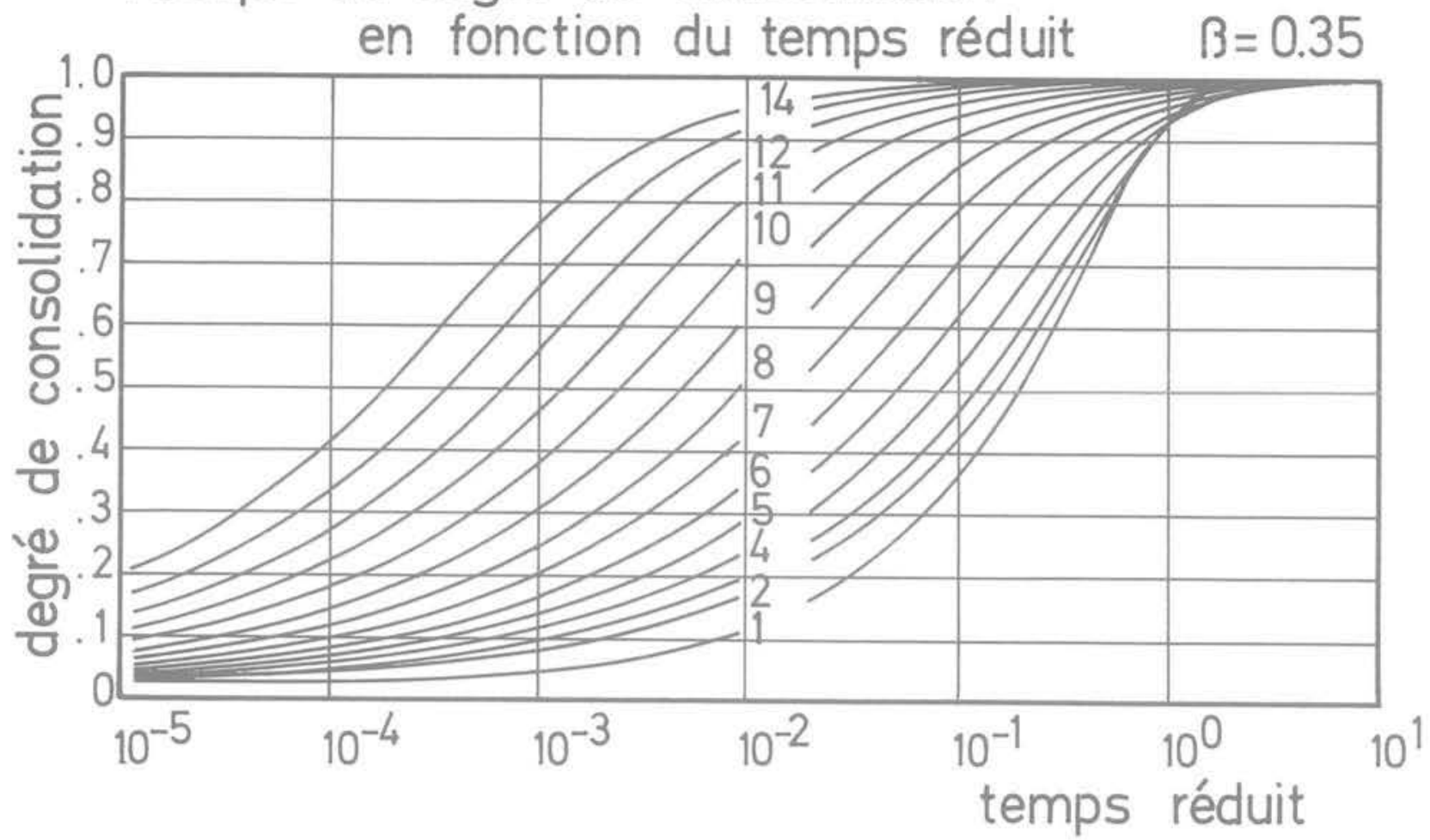

Abaque du degré de tassement

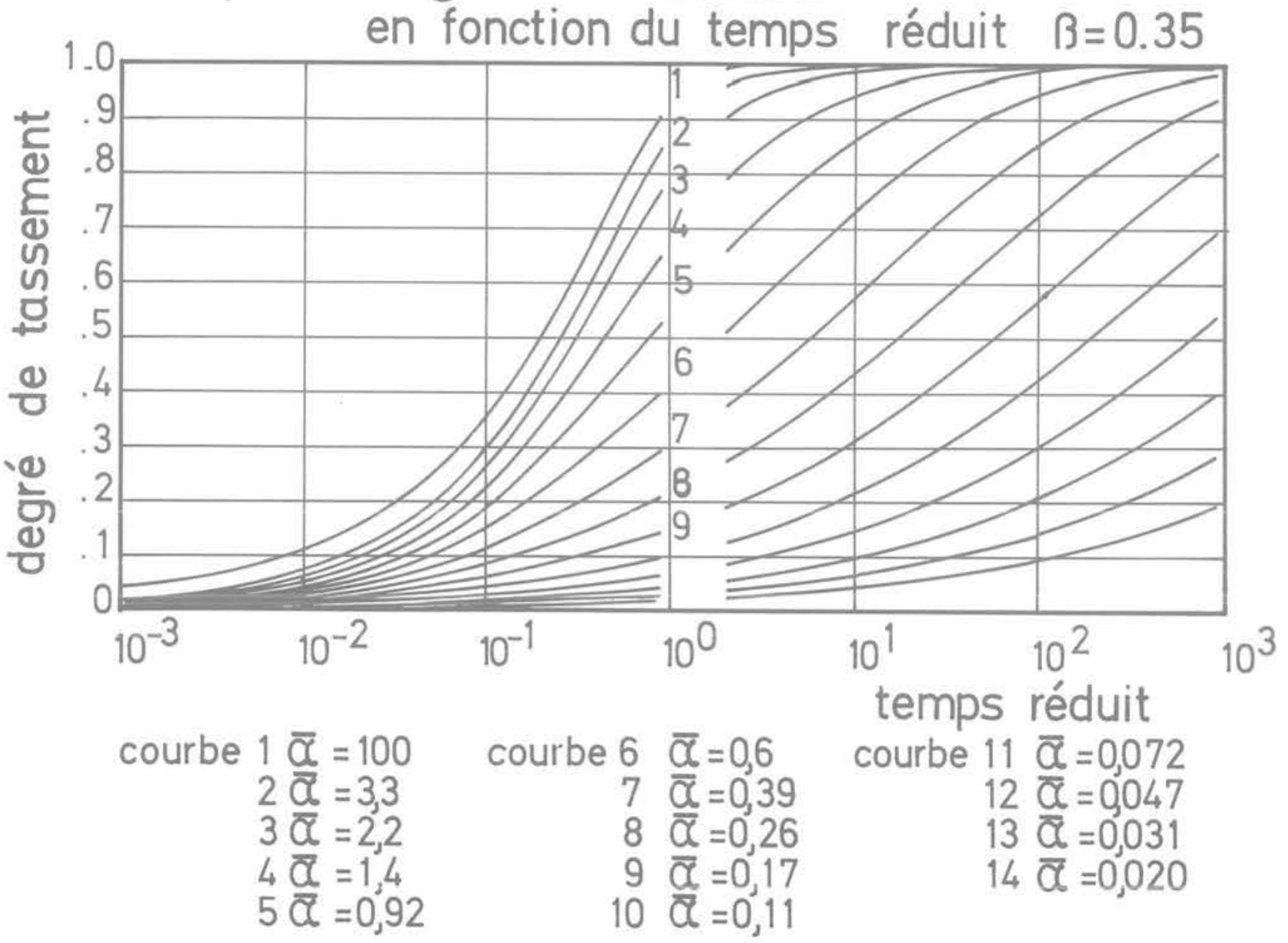

Fig. 12 Abaques des degrés de consolidation et de tassement de sols dont la viscosité est grande $(\beta=0,35)$ 
application aux cas précédents ne peut être envisagée que si le module de compressibilité œdométrique est défini à partir de la déformation que provoque l'incrément de charge au bout d'un temps infini.

Les valeurs courantes de $\bar{\alpha}$ correspondent à une évolution du degré de consolidation en fonction du temps réduit, plus rapide que ne le prévoit la solution classique. Par contre, celle du degré de tassement est plus lente. La courbe représentative de cette solution sert de limite aux deux faisceaux de courbes, l'un étant situé au-dessus et l'autre au-dessous. L'écart entre cette courbe limite et une courbe quelconque, qui peut être considérable, est d'autant plus grand que $\bar{\alpha}$ est plus petit, donc entre autre que le coefficient d'échelle est plus petit. Mais le paramètre $\beta$, qui est égal à la puissance à laquelle il est porté, tempère cette influence lorsqu'il est petit. Une petite valeur de $\beta$ signifie une vitesse de fluage initiale du squelette qui est grande, et qui ne se rencontre que pour des sols compressibles, soumis à de fortes charges. On peut donc dire que la consolidation de ces sols, quelles que soient les conditions de drainage, présente une bonne conformité aux prévisions de la théorie de K. Terzaghi. Sur les abaques de la figure 11, les faisceaux de courbes sont beaucoup plus étroits que sur la figure 12 qui représente la consolidation de sols dont la viscosité est très grande.

Sur cette dernière figure il apparaît bien, que l'essentiel de la consolidation (jusqu'à un degré de $95 \%$ ) se développe plus vite pour les sols à squelette visqueux, mais l'achèvement de cette consolidation correspond à un temps réduit plus long que d'après la théorique classique. (Les courbes du faisceau passent sous celle de la solution classique.) Cette remarque concorde avec les résultats expérimentaux et les calculs relatifs aux échantillons soumis à un taux d'accroissement de la charge de 1,20; les surpressions interstitielles sont plus petites que lorsque ce taux est égal à 2,15, mais l'achèvement de leur dissipation se produit au bout d'un temps réel plus long.

\section{Utilisation pratique des abaques}

Les paramètres dont on a besoin sont déterminés grâce à un essai œdométrique sur un échantillon représentatif du sol de fondation. Après reconsolidation sous le poids des terres en place et mesure de la perméabilité initiale, l'échantillon est consolidé sous un incrément de charge $\Delta \sigma$ égal à celui provoqué par l'ouvrage. Les tassements sont mesurés pendant 3 jours au moins, de façon à faire apparaître une fraction importante de la consolidation secondaire. La perméabilité est mesurée une seconde fois à la fin de l'essai.

Le coefficient de perméabilité retenu est égal à la moyenne des deux valeurs trouvées.

La déformation finale $\varepsilon_{\infty}$ du sol est calculée par la formule (28) (Félix, $1980 \mathrm{c}$ ) :

$$
\varepsilon_{\infty}=\varepsilon_{1}+\left(\varepsilon_{n}-\varepsilon_{1}\right) \frac{\lg 100 \text { ans }-\lg t_{1}}{\lg t_{n}-\lg t_{1}} .
$$

Elle est extrapolée suivant une loi logarithmique du temps, déduite de la première déformation de consolidation secondaire $\varepsilon_{1}$ mesurée au temps $t_{1}$, et de la dernière $\varepsilon_{n}$ mesurée au temps $t_{n}$. La connaissance de $\varepsilon_{\infty}$ permet de déterminer,

d'une part, le module de déformation œdométrique à long terme :
$E_{t 1}=\frac{\Delta \sigma}{\varepsilon_{\infty}}$ et le coefficient de consolidation fluage $C_{v f}=\frac{k E_{t f}}{\gamma_{w}}$, et, d'autre part, les paramètres rhéologiques $\alpha$ et $\beta$ du squelette intergranulaire. Pour cela on substitue au temps $\mathrm{t}$ la fonction In $\mathrm{t}$ et à la déformation $\varepsilon$ l'expression $\ln \left[\ln \frac{\varepsilon_{\infty}}{\varepsilon_{\infty}-\varepsilon}\right]$. La droite des moindres carrés qui corrèle les mesures transformées $\ln \left[\ln \frac{\varepsilon_{\infty}}{\varepsilon_{\infty}-\varepsilon_{i}}\right]$ en fonction de In $t_{i}$, a une pente égale à $\beta$ et une ordonnée à l'origine égale à In $\alpha$; (Félix, 1980 c). Parmi les valeurs choisies pour tracer les abaques, la plus proche de $\beta$ est retenue, à laquelle il peut être intéressant d'associer une valeur corrigée du paramètre $\alpha$ (Félix, 1980 b) :

$$
\alpha=\frac{1}{n} \frac{\sum_{i=1}^{n} \ln \frac{\varepsilon_{\infty}}{\varepsilon_{\infty}-\varepsilon_{i}}}{\sum_{i=1}^{n} t_{i}^{\beta}} .
$$

Sur cet abaque, les 2 courbes dont les paramètres $\bar{\alpha}$ encadrent la valeur retenue $\bar{\alpha}=\alpha\left[\frac{h^{2}}{C_{v t}}\right]^{\beta}$ permettent, par extrapolation linéaire suivant le logarithme de $\bar{\alpha}$, de déterminer le temps réduit correspondant à un degré de déformation ou de consolidation donné. Le temps réel correspondant est lié au temps réduit par la relation (29) :

$$
\mathrm{t}=\mathrm{T} \frac{\mathrm{h}^{2}}{\mathrm{C}_{\mathrm{vt}}} \text {. }
$$

A titre d'exemple, les temps nécessaires pour que les quatre échantillons de longueurs de drainage différentes, soumis au premier palier de $40 \mathrm{kPa}$, ainsi que le sol de fondation du remblai $\mathrm{B}$, présentent une déformation de $5 \%$, ont été déterminés par les abaques et par le programme de calcul Hydroflu. La solution fournie par la théorie classique a été également reportée sur le tableau 4. Le module œdométrique utilisé dans cette théorie est calculé sur la base de la déformation après 24 heures de l'échantillon dont la longueur de drainage est égale à $10 \mathrm{~mm}$; soit $10 \%$. La déformation de $5 \%$ correspond donc à un degré de consolidation égal à $50 \%$. A titre de comparaison, les temps nécessaires pour atteindre ce degré, d'après les abaques et d'après le programme Hydroflu, sont également indiqués sur le tableau 4.

Les résultats de ces deux dernières méthodes de calcul présentent peu de divergences; le fluage du squelette apparaît bien comme le facteur déterminant, qu'il convenait d'inclure dans les hypothèses. Le temps réel mesuré correspondant à $5 \%$ de déformation est largement sous-estimé par la théorie classique, pour les longueurs de drainage égales à celle des échantillons œdométriques. Il est surestimé pour le sol de fondation du remblai $B$. Pour une telle longueur de drainage, la forme des équations de la théorie proposée s'identifie à celle de la théorie classique, mais la divergence provient du module de compressibilité œdométrique. Dans tous les cas, les abaques du fluage et la consolidation fournissent un meilleur résultat.

Quant aux temps nécessaires pour atteindre le degré de consolidation de $50 \%$, qui ne peuvent être comparés à aucune mesure, ils sont beaucoup plus courts d'après le programme Hydroflu et les abaques que d'après la théorie classique, pour les longueurs de drainage égales à celles des échantillons œdométri- 


\begin{tabular}{l|c|c|c|c|c}
\hline longueur de drainage & $5 \mathrm{~mm}$ & $10 \mathrm{~mm}$ & $20 \mathrm{~mm}$ & $40 \mathrm{~mm}$ & $4,5 \mathrm{~m}$ \\
\hline degré de consolidation $\mathrm{U}=50 \%$ abaque & $1,4 \mathrm{mn}$ & $6,6 \mathrm{mn}$ & $31 \mathrm{mn}$ & $140 \mathrm{mn}$ & 6 ans \\
\hline degré de consolidation $U=50 \%$ Hydroflu & $1,3 \mathrm{mn}$ & $6,5 \mathrm{mn}$ & $33 \mathrm{mn}$ & $150 \mathrm{mn}$ & 9,5 ans \\
\hline déformation $\varepsilon=5 \%-\mathrm{U}=50 \%$ Terzaghi & $3,1 \mathrm{mn}$ & $12,5 \mathrm{mn}$ & $50 \mathrm{mn}$ & $200 \mathrm{mn}$ & 4,8 ans \\
\hline déformation $\varepsilon=5 \%$ mesure & $10 \mathrm{mn}$ & $18 \mathrm{mn}$ & $65 \mathrm{mn}$ & $200 \mathrm{mn}$ & 1 an \\
\hline déformation $\varepsilon=5 \%$ abaque & $7,7 \mathrm{mn}$ & $23 \mathrm{mn}$ & $70 \mathrm{mn}$ & $250 \mathrm{mn}$ & 3,4 ans \\
\hline déformation $\varepsilon=5 \%$ Hydroflu & $7,7 \mathrm{mn}$ & $22 \mathrm{mn}$ & $72 \mathrm{mn}$ & $240 \mathrm{mn}$ & 2,7 ans \\
\hline Tableau 4
\end{tabular}

Tableau 4 Comparaison entre les resultats expérimentaux d'une part et les calculs par la théorie de $K$. Terzaghi, Hydroflu et les abaques de la consolidation et du fluage d'autre part

ques. Ceci confirme que la viscosité du squelette, toutes choses étant égales par ailleurs, accélère la dissipation des surpressions interstitielles, tout au moins dans la phase initiale de la consolidation hydrodynamique.

\section{Conclusion}

Le fluage du squelette intergranulaire est un facteur capital intervenant dans la consolidation des sols. II détermine la phase secondaire et exerce une grande influence sur l'évolution de la phase hydrodynamique. La théorie montre que, si le sol est de plus imparfaitement saturé, les surpressions interstitielles commencent par croître vers une valeur maximale inférieure à la charge appliquée, mais cette caractéristique perd de son influence à mesure que la dissipation des surpressions par écoulement du fluide interstitiel se développe.

$\mathrm{Si}$, à l'exception des hypothèses relatives au fluage du squelette, toutes les autres modifications apportées à la théorie de la consolidation de K. Terzaghi sont abandonnées, les solutions peuvent être présentées sous forme d'abaques. Les données nécessaires à leur utilisation, sont obtenues grâce à un essai de compressibilité-perméabilité à l'œdomètre. Les abaques montrent que la viscosité du squelette est d'autant plus influente, que la longueur de drainage est plus petite. Toute chose étant égale par ailleurs, sa prise en compte diminue en général les surpressions interstitielles. La théorie classique apparaît comme un cas particulier qui concerne les monocouches particulièrement épais et compressibles.

\section{Références bibliographiques}

Abelev M. Yu., Tsytovich N. A. (1964), "Questions sur l'application de la théorie de la consolidation hydrodynamique aux sols argileux saturés et très compressibles", journal Soil Mechanics and Foundation Engineering, traduction anglaise du journal Osnovaniya Fundamenty i Mekhanika Gruntov, mai-juin 1964, $n^{\circ} 3$, p. 158-163.

Felix B. (1980) a. « Le fluage des sols argileux, étude bibliographique», Rapport de Recherche LPC, n 93 , Paris.

Felix B. (1980) b. "Fluage et consolidation unidimensionnelle des sols argileuxn, Rapport de Recherche LPC, $n^{\circ} 94$, Paris.
Felix B. (1980) c. «Étude théorique et expérimentale du fluage unidimensionnel des sols argileux $»$, Revue française de Géotechnique, n¹3, novembre 1980.

Fredlund D.G. (1976). "Density and compressibility characteristics of air-water mixtures $*$, Canadian geotechnical Journal, vol. $13, n^{\circ} 4$, novembre 1976 , p. 386396.

Magnan J. P., Dang M. T. (1977), «Étude théorique et expérimentale de la compressibilité du fluide interstitiel dans un sol argileux presque saturé ", bulletin de liaison des Laboratoires des Ponts et Chaussées, numéro spécial VIF, "mécanique des sols", décembre 1977, p. 129-137.

Magnan J. P. et al (1979). «Étude numérique de la consolidation unidimensionnelle en tenant compte des variations de la perméabilité et de la compressibilité du sol, du fluage et de la non saturation », bulletin de liaison des Laboratoires des Ponts et Chaussées, $n^{\circ} 103$, septembre-octobre 1979 , p. 83-94.

Meschyan S. R. (1967). Le fluage des sols argileux (en russe) édition de l'Académie des Sciences de la RSS d'Arménie, 1967, 318 pages.

Mesri G., Rokhsar A. (1974). «Theory of consolidation of clays", Journal of the Geotechnical Engineering Division, Proceedings of the A.S.C.E. vol. 100, GT 8, août 1974 , p. $889-904$.

Schuurman I.E. (1966). «The compressibility of an air/water mixture and a theoretical relation between the air and water pressures ", Geotechnique, vol. XVI, $n^{\circ} 4$, décembre 1966, p. 269-281.

Tavenas F. et al (1978). «Pressions interstitielles développées dans l'argile de fondation des remblais, deuxième partie; comportement généralisé ", bulletin de liaison des Laboratoires des Ponts et Chaussées, $n^{\circ} 98$, septembre-octobre 1978 , p. $53-67$.

Ter Martirosyan Z. G. (1967). «Chapitre V : Quelques problèmes de consolidation des sols argileux prenant en compte simultanément le fluage du squelette et la compressibilité du liquide interstitiel ». Prévision de la vitesse de tassement des sols de fondation des ouvrages (en russe). Édition litératury po stroitel' stvu, Moscou 1967, p. 115-156.

Terzaghi K. (1923). "Calcul de la perméabilité de l'argile à partir de la durée de la consolidation hydrodynamique» Sitzungsberichte Matematisch Naturwissenschaftliche Klasse, 1923, Vienne Akademie der Wissenschaften, part. 2 a, 132, 3/4, p. 125-138.

Thomann G. (1972). "Résolution de l'équation de la consolidation unidimensionnelle par la méthode des différences finies $\%$, bulletin de liaison des Laboratoires des Ponts et Chaussées, $n^{\circ}$ 59, mai-juin 1972, p. 57-64. 
